\title{
A direct computation of the cohomology of the braces operad
}

Dolgushev, Vasily ; Willwacher, Thomas

\begin{abstract}
We give a self-contained and purely combinatorial proof of the well-known fact that the cohomology of the braces operad is the operad Ger governing Gerstenhaber algebras.
\end{abstract}

DOI: https://doi.org/10.1515/forum-2016-0123

Posted at the Zurich Open Repository and Archive, University of Zurich ZORA URL: https://doi.org/10.5167/uzh-207522

Journal Article

Published Version

Originally published at:

Dolgushev, Vasily; Willwacher, Thomas (2017). A direct computation of the cohomology of the braces operad. Forum Mathematicum, 29(2):465-488.

DOI: https://doi.org/10.1515/forum-2016-0123 


\title{
Research Article
}

\section{Vasily Dolgushev* and Thomas Willwacher \\ A direct computation of the cohomology of the braces operad}

DOI: $10.1515 /$ forum-2016-0123

Received May 28, 2016

\begin{abstract}
We give a self-contained and purely combinatorial proof of the well-known fact that the cohomology of the braces operad is the operad Ger governing Gerstenhaber algebras.
\end{abstract}

Keywords: Algebraic operads, homotopy algebras, the Deligne conjecture on Hochschild cochains

MSC 2010: 18D50, 18G55, 55P10

Communicated by: Frederick R. Cohen

\section{Introduction}

It is a well-known fact [7] that the Hochschild cohomology of an associative (or $A_{\infty}$ ) algebra $A$ carries the structure of a Gerstenhaber algebra. In 1993, P. Deligne [3] asked whether this Gerstenhaber algebra structure is induced by an action of some version of the chains operad of the little disks operad on the Hochschild cochain complex $C^{\bullet}(A, A)$ of $A$. This question became known as the Deligne conjecture and was answered affirmatively by various authors including C. Berger and B. Fresse [1], R. M. Kaufmann [11, 12], M. Kontsevich and Y. Soibelman [15], J. E. McClure and J. H. Smith [19], and D. Tamarkin [22, 24].

A key role in the proof of the Deligne conjecture is played by the braces operad $\mathrm{Br}$, which encodes a set of natural operations on the Hochschild cochain complex $C^{*}(A, A)$ of any $A_{\infty}$ algebra $A$. In the form used here, this differential graded (dg) operad was introduced by Kontsevich and Soibelman [15], where it is called the "minimal operad". Its (quasi-isomorphic) variant (see also [10, Section 3.12] for the description of the map between the associative and $A_{\infty}$ version) for associative algebras was considered by McClure and Smith [20], and both constructions go back to earlier work of Getzler [8] (cf. also [9]).

The goal of this note is to give a purely combinatorial proof of the fact that the operad $H^{*}(\mathrm{Br})$ is isomorphic to the operad Ger which governs Gerstenhaber algebras.

Concretely, the $n$-th space $\operatorname{Br}(n)$ of the braces operad is spanned - in this note, the ground field $\mathbb{K}$ is any field of characteristic zero - by planar rooted trees (called brace trees) with $n$ vertices labeled by $1,2, \ldots, n$ and some (possibly zero) number of unlabeled (or neutral) vertices. The grading on $\operatorname{Br}(n)$ is obtained by declaring that each non-root edge carries degree -1 and each neural vertex carries degree 2 . In pictures, white circles with inscribed numbers denote labeled vertices and black circles denote neutral vertices (we tacitly assume that every neutral vertex has at least two children). Several examples of brace trees are shown in Figure 1.

Thus the brace trees $T_{1-2}$ and $T_{2-1}$ have degree -1 while the brace trees $T_{\cup}$ and $T_{\cup}^{\mathrm{opp}}$ have degree 0 .

\footnotetext{
*Corresponding author: Vasily Dolgushev: Department of Mathematics, Temple University, Wachman Hall , Rm. 638, 1805 N. Broad St., Philadelphia, PA, 19122 USA, e-mail: vald@temple.edu

Thomas Willwacher: Institute of Mathematics, University of Zürich, Winterthurerstraße 190, 8057 Zürich, Switzerland, e-mail: thomas.willwacher@math.uzh.ch
} 


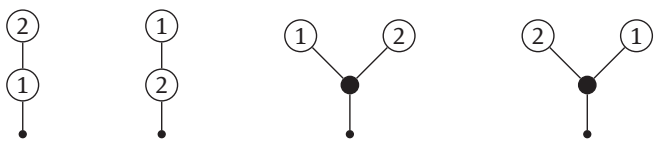

Figure 1. The brace trees $T_{1-2}, T_{2-1}, T_{\cup}$ and $T_{\cup}^{\text {opp }}$ from left to right, respectively.

The differential $\delta(T)$ of a brace tree $T$ is defined by the formula

$$
\delta(T):=\sum_{j=1}^{n} \delta_{j}(T)+\sum_{v} \delta_{v}(T),
$$

where the second sum is over all neutral vertices and the operations $\delta_{j}, \delta_{v}$ are defined graphically as follows:

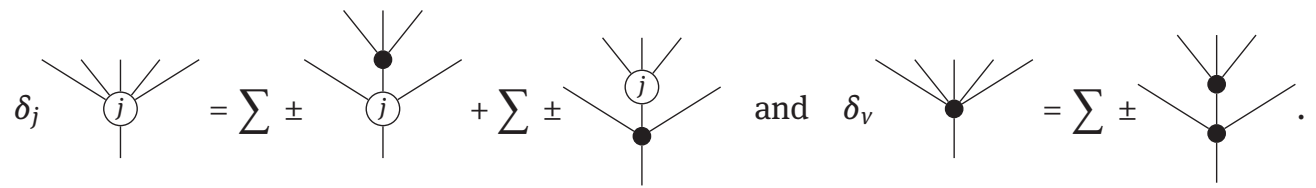

The operadic multiplications are defined in terms of natural combinatorial operations with planar trees. For more details we refer the reader to Section 3 of this note or [5, Sections 7-9].

The dg operad $\mathrm{Br}$ acts on the Hochschild cochain complex $C^{*}(A, A)$ of an $A_{\infty}$-algebra. The detailed description of this action is given in [5, Appendix B]. For example, for $P_{1}, P_{2} \in C^{*}(A, A)$, the cochain $T_{\cup}\left(P_{1}, P_{2}\right)$ (resp. $T_{1-2}\left(P_{1}, P_{2}\right)+T_{2-1}\left(P_{1}, P_{2}\right)$ ) coincides (up to a sign factor) with the cup product $P_{1} \cup P_{2}$ (resp. the Gerstenhaber bracket $\left.\left[P_{1}, P_{2}\right]_{G}\right)$.

Let us recall (see Appendix A) that the $S_{2}$-invariant $\delta$-cocycle

$$
T_{\left\{a_{1}, a_{2}\right\}}:=T_{1-2}+T_{2-1}
$$

satisfies the Jacobi relation

$$
T_{\left\{a_{1}, a_{2}\right\}}{ }^{\circ} T_{\left\{a_{1}, a_{2}\right\}}+(1,2,3)\left(T_{\left\{a_{1}, a_{2}\right\}}{ }^{\circ} T_{\left\{a_{1}, a_{2}\right\}}\right)+(1,3,2)\left(T_{\left\{a_{1}, a_{2}\right\}}{ }^{\circ} T_{\left\{a_{1}, a_{2}\right\}}\right)=0 .
$$

Therefore, we have a natural operad map

$$
\mathrm{j}: \Lambda \mathrm{Lie} \rightarrow \mathrm{Br}
$$

from the shifted version $\Lambda$ Lie of the operad Lie to the dg operad $\mathrm{Br}$.

It is easy to check that the cocycle $T_{\cup}$ satisfies the associativity relation up to homotopy

$$
T_{\cup} \circ_{1} T_{\cup}-T_{\cup} \circ_{2} T_{\cup} \in \operatorname{Im}(\delta)
$$

and the difference

$$
T_{\cup}-T_{\cup}^{\mathrm{opp}}
$$

is $\delta$-exact.

Therefore, we have a natural operad map

$$
\mathrm{Com} \rightarrow H^{\bullet}(\mathrm{Br})
$$

which sends the generator of Com to the cohomology class of the $\delta$-cocycle:

$$
T_{a_{1} a_{2}}:=\frac{1}{2}\left(T_{\cup}+T_{\cup}^{\mathrm{opp}}\right) \text {. }
$$

It is also easy to check (see Appendix A) that the $\delta$-cocycles $T_{a_{1} a_{2}}$ and $T_{\left\{a_{1}, a_{2}\right\}}$ satisfy the Leibniz rule up to homotopy, i.e.

$$
T_{\left\{a_{1}, a_{2}\right\} \circ_{2}} T_{a_{1} a_{2}}-T_{a_{1} a_{2}} \circ_{1} T_{\left\{a_{1}, a_{2}\right\}}-(1,2)\left(T_{a_{1} a_{2} \circ 2} T_{\left\{a_{1}, a_{2}\right\}}\right) \in \operatorname{Im}(\delta) .
$$

Thus, combining the maps (1.2) and (1.3), we get an operad map

$$
\text { Ger } \rightarrow H^{*}(\mathrm{Br}) \text {. }
$$

In this note, we give a self-contained combinatorial proof of the following theorem:

Theorem 1.1. The map (1.5) is an isomorphism of operads. 
This theorem is a shadow of the very deep statement which says that the dg operad $\mathrm{Br}$ is weakly equivalent to the operad Ger. The proof of the latter statement involves a solution of the Deligne conjecture and the formality of the dg operad $C_{-}$. $\left(E_{2}, \mathbb{K}\right)$, where $E_{2}$ denotes the topological operad of little dikcs [23]. One possible proof (see [23]) of the formality of $C_{-}$. $\left(E_{2}, \mathbb{K}\right)$ involves the use of Drinfeld's associator [6] and another possible proof (see [14, Section 3.3], [16]) involves the use of a configuration space integral. Although Theorem 1.1 does not imply the formality of the operad $\mathrm{Br}$, it is amazing that it can be proved in a purely combinatorial way which bypasses the use of compactified configuration spaces.

We should remark that various topological proofs of Theorem 1.1 were given earlier. One such proof is sketched, for example, in [15, Theorem 4], and another proof may be extracted from [20], together with a small computation. Finally, a third proof is described in [11, 13].

Let us also remark that our proof admits a straightforward generalization to the higher versions of the braces operads $\mathrm{Br}_{n+1}$ acting naturally on the deformation complexes of $n$-algebras, cf. [2, Section 4].

Remark 1.2. There is an amazing combinatorial similarity between the dg operad $\mathrm{Br}$ and the dg operad Graphs [14, Section 3.3], [21, Section 3]. The latter dg operad is "assembled from" graphs of certain kind with some additional data and the former dg operad is "assembled from" rooted planar trees (also with some additional data). Both dg operads are formal. In fact, both dg operads are weakly equivalent to the same operad Ger. However, while the proof of formality for Graphs involves only elementary homological algebra [14, Section 3.3.4], the proof of formality for Br requires a "very heavy hammer".

\subsection{The organization of the paper and the outline of the proof of Theorem 1.1}

In Section 2, we fix some necessary notational conventions. In Section 3, we give a more detailed description of the dg operad Br. In Section 4, we formulate and prove a more refined version of Theorem 1.1 (see Theorem 4.2). Appendix A is devoted to the proof of the fact that the vectors (1.1) and (1.4) of Br satisfy the Gerstenhaber relations up to homotopy. Finally, Appendix B is devoted to a proof of a technical statement about the spectral sequence used in Section 4.

Using a standard basis for the space $\operatorname{Ger}(n)$ and vectors (1.1) and (1.4), we define a map of dg collections $\Psi:$ Ger $\rightarrow$ Br (see Section 4.1).

Claim A.1 from Appendix A implies that the map

$$
H^{\bullet}(\Psi): \text { Ger } \rightarrow H^{*}(\mathrm{Br})
$$

is compatible with the operad structure.

To prove that the map $H^{\bullet}(\Psi)$ induces an isomorphism of operads, we proceed by induction on the arity $n$. Since the base of the induction $n=1$ is obvious, we assume that $\Psi$ induces isomorphisms

$$
H^{\bullet}(\operatorname{Br}(j)) \cong \operatorname{Ger}(j)
$$

for all $1 \leq j \leq n-1$, we split the graded vector space into the direct sum

$$
\operatorname{Br}(n)=V_{\mathrm{o}}(n) \stackrel{\delta_{0}}{\delta_{1}} \begin{gathered}
\delta_{0} \\
\oplus V_{0}(n),
\end{gathered}
$$

where $V_{\bullet}(n)$ is the subspace of $\operatorname{Br}(n)$ spanned by brace trees whose lowest non-root vertex is neutral and $V_{\circ}(n)$ is the subspace of $\operatorname{Br}(n)$ spanned by brace trees whose lowest non-root vertex is labeled. The arrows in the above formula indicate the non-zero components of the differential.

It is clear that

- both $V_{\circ}(n)$ and $V$. $(n)$ may be considered as cochain complexes with the differential $\delta_{0}$.

- $\delta_{1}$ induces a map

$$
H^{\bullet}\left(\delta_{1}\right): H^{\bullet}\left(V_{\circ}(n), \delta_{0}\right) \rightarrow H^{\bullet}\left(V_{\bullet}(n), \delta_{0}\right),
$$

- $H^{\bullet}(\operatorname{Br}(n)) \cong\left(\operatorname{ker} H^{\bullet}\left(\delta_{1}\right)\right) \oplus\left(\operatorname{coker} H^{\bullet}\left(\delta_{1}\right)\right)$. 
In Section 4.3, we prove that $H^{\bullet}\left(V_{\circ}(n), \delta_{0}\right)$ is isomorphic to $\mathbf{s}^{n-1} \mathbb{K}\left[S_{n}\right]$ as the $S_{n}$-module and show that the cohomology class corresponding to $\lambda \in S_{n}$ is represented by the brace tree $T_{\lambda}^{n}$ depicted in Figure 12 .

In Section 4.4, we establish an isomorphism of $S_{n}$-modules

$$
H^{\bullet}\left(V_{\bullet}(n), \delta_{0}\right) \cong \operatorname{Com} \odot \Lambda \operatorname{Lie}(n) / \Lambda \operatorname{Lie}(n) \oplus \mathbf{S}(\Lambda \operatorname{Com} \odot \Lambda \operatorname{Lie}(n) / \Lambda \operatorname{Lie}(n)),
$$

where $\odot$ denotes the plethysm of collections.

This is done by filtering $V_{\bullet}(n)$ by the number of children of the lowest non-root vertex and analyzing the corresponding spectral sequence. The main technical statement

$$
E_{\infty}\left(V \cdot(n), \delta_{0}\right)=E_{2}\left(V \cdot(n), \delta_{0}\right)
$$

about this spectral sequence is proved separately (see Lemma B.4) in Appendix B.

In Section 4.5, we prove a technical statement about the dual version of the map (1.6). Finally, in Section 4.6, we use this technical statement and the results of the previous sections to complete the proof of Theorem 4.2.

\section{Notation}

We work over a ground field $\mathbb{K}$ of characteristic 0 . For a set $X$ we denote by $\operatorname{span}_{\mathbb{K}}(X)$ the $\mathbb{K}$-vector space of finite linear combinations of elements in $X$. We denote by $\mathbf{s}$ (resp. $\mathbf{s}^{-1}$ ) the operation of suspension (resp. desuspension) for graded or differential graded ( $d g$ for short) $\mathbb{K}$ vector spaces. The notation $|v|$ is reserved for the degree of a homogeneous vector $v$ in a (differential) graded vector space.

By a collection we mean a sequence $\{P(n)\}_{n \geq 0}$ of dg vector spaces with a right action of the symmetric group $S_{n}$. The category of collections carries a natural monoidal structure, the plethysm operation $\odot$, see, e.g., [5, equation (5.1)].

We will freely use the language of operads. A good introduction is provided in textbook [18]. The notation Lie (resp. As, Com, Ger) is used for the operad governing Lie algebras (resp. associative, commutative or Gerstenhaber algebras without unit). Dually, the notation coLie (resp. coAs, coCom) is reserved for the cooperad governing Lie coalgebras (resp. coassociative coalgebras without counit, cocommutative (and coassociative) coalgebras without counit).

For an operad $\mathcal{O}($ resp. a cooperad $\mathcal{C})$ and a cochain complex $V$, we denote by $\mathcal{O}(V)$ (resp. $\mathcal{C}(V))$ the free $\mathcal{O}$-algebra (resp. cofree $\mathcal{C}$-coalgebra).

For an operad (resp. a cooperad) $P$ we denote by $\Lambda P$ the operad (resp. the cooperad) with the spaces of $n$-ary operations:

$$
\Lambda P(n)=\mathbf{s}^{1-n} P(n) \otimes \operatorname{sgn}_{n},
$$

where $\operatorname{sgn}_{n}$ denotes the sign representation of $S_{n}$.

For an operad $\mathcal{O}$ and degree 0 auxiliary variables $a_{1}, a_{2}, \ldots, a_{n}, \mathcal{O}(n)$ is naturally identified with the subspace of the free $\mathcal{O}$-algebra

$$
\mathcal{O}\left(\operatorname{span}_{\mathbb{K}}\left(a_{1}, a_{2}, \ldots, a_{n}\right)\right)
$$

spanned by $\mathcal{O}$-monomials in which each variable from the set $\left\{a_{1}, a_{2}, \ldots, a_{n}\right\}$ appears exactly once. We often use this identification in this paper. For example, the vector space Ger(2) of the operad Ger is spanned by the degree zero vector $a_{1} a_{2}$ and the degree -1 vector $\left\{a_{1}, a_{2}\right\}$. The commutative (and associative) multiplication on a Gerstenhaber algebra $V$ comes from the vector $a_{1} a_{2} \in \operatorname{Ger}(2)$ and the odd Lie bracket $\{\cdot, \cdot\}$ on $V$ comes from the vector $\left\{a_{1}, a_{2}\right\} \in \operatorname{Ger}(2)$. Similarly, the space $\Lambda \operatorname{Lie}(n)$ of the suboperad $\Lambda$ Lie $\subset$ Ger is spanned by $\Lambda$ Lie-monomials in $a_{1}, a_{2}, \ldots, a_{n}$ in which each variable from the set $\left\{a_{1}, a_{2}, \ldots, a_{n}\right\}$ appears exactly once. For example, $\Lambda \operatorname{Lie}(2)$ is spanned by the vector $\left\{a_{1}, a_{2}\right\}$ and $\Lambda \operatorname{Lie}(3)$ is spanned by the vectors $\left\{\left\{a_{1}, a_{2}\right\}, a_{3}\right\}$ and $\left\{\left\{a_{1}, a_{3}\right\}, a_{2}\right\}$.

Let us recall [4, Section 2, p. 32] that the set of edges of any planar tree $T$ is equipped with the natural total order. We use this total order to determine sign factors in various computations related to the operad $\mathrm{Br}$. 


\section{Brace trees, a reminder of the dg operad $\mathrm{Br}$}

Let us recall that a brace tree is a rooted planar tree having two kinds of non-root vertices:

- labeled vertices, numbered $\{1,2,3, \ldots\}$,

- an arbitrary number of unlabeled neutral vertices.

In addition, one requires that each neutral vertex has at least two children. For example, Figure 2 shows a brace tree $T$ with six labeled vertices. In pictures, white circles with inscribed numbers denote labeled vertices, black circles denote neutral vertices, and the small black node (at the bottom) denotes the root.

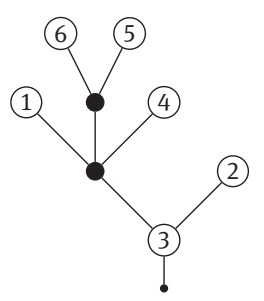

Figure 2. An example of a brace tree.

Let $\operatorname{Br}(n)$ be the linear span of the set of brace trees with exactly $n$ labeled vertices. The $\mathbb{Z}$-grading on $\operatorname{Br}(n)$ is given by declaring that each brace tree has the degree

$$
2 \times \text { number of neutral vertices }- \text { number of non-root edges. }
$$

For example, the brace tree shown in Figure 2 has degree -3 .

Let $T$ be a brace tree with $n$ labeled vertices, $j$ be a number between 1 and $n$, and $v$ be a neutral vertex of $T$ (if $T$ has one). To recall the definition of the differential $\delta$ on $\operatorname{Br}(n)$, we introduce these three vectors

$$
\delta_{j}^{\prime}(T), \quad \delta_{j}^{\prime \prime}(T), \quad \delta_{v}(T)
$$

in $\operatorname{Br}(n)$. The vector $\delta_{j}^{\prime}(T)$ (resp. $\delta_{j}^{\prime \prime}(T)$ ) is obtained from $T$ in the three steps:

- First, we replace vertex $j$ by the left most branch in Figure 3 (resp. the middle branch in Figure 3).

- Second, we reconnect the edges which originated from vertex $j$ to this branch in all ways compatible with the planar structure.

- Finally, we discard all brace trees which have a neutral vertex of valency $<3$. Similarly, the vector $\delta_{v}(T)$ is obtained from $T$ in the three steps:

- First, we replace the neutral vertex $v$ with the right most branch in Figure 3.

- Second, we reconnect the edges which originated from vertex $v$ to this branch in all ways compatible with the planar structure.

- Finally, we discard all brace trees which have a neutral vertex of valency $<3$.

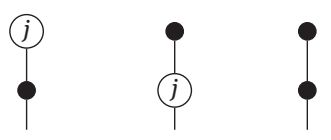

Figure 3. The branches appearing in the definition of the differential.

The differential $\delta(T)$ of a brace tree $T \in \operatorname{Br}(n)$ is the sum over all labeled and all neutral vertices

$$
\delta(T)=\sum_{j=1}^{n}\left(\delta_{j}^{\prime}(T)+\delta_{j}^{\prime \prime}(T)\right)+\sum_{v} \delta_{v}(T) .
$$

The signs in the sums $\delta_{j}^{\prime}(T), \delta_{j}^{\prime \prime}(T)$, and $\delta_{v}(T)$ are determined by treating non-root edges as "anti-commuting variables.” 

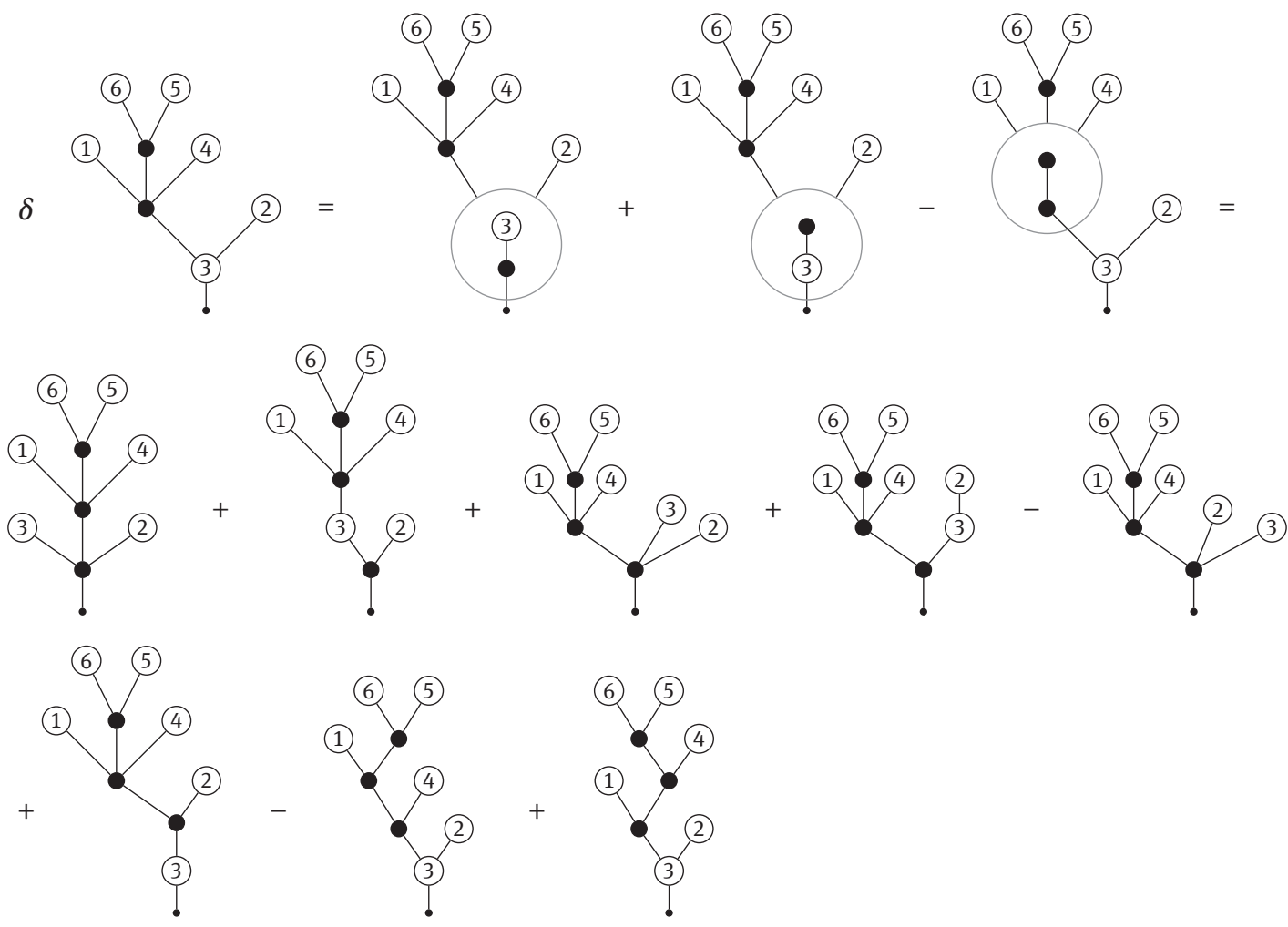

Figure 4. Example of computing $\delta(T)$.

For example, for the brace tree $T$ shown in Figure 2, the computation of the differential is shown in Figure 4. The sign " "-" in front of the right most term in the first line appears to due to the fact the additional edge has to "move behind" the edge originating from vertex 3 . The signs in front of the first four terms in the second line are pluses since the branch which originates at vertex 3 of $T$ has the even number of edges. The sign "-" in front of the right most term in the second line appears because the edge adjacent to vertex 2 "moves ahead" of the additional edge. The signs in the third line are obtained in the similar fashion.

Let us observe that, since we discard brace trees with at least one neutral vertex of valency $\leq 2$, we have

$$
\delta_{j}^{\prime}(T)=\delta_{j}^{\prime \prime}(T)=0 \quad \text { and } \quad \delta_{v}(T)=0
$$

if vertex $j$ is univalent and (neutral) vertex $v$ is trivalent. Also, if vertex $j$ is bivalent, then $\delta_{j}^{\prime \prime}(T)=0$.

A simple example of the computation of an elementary insertion is shown in Figure 5. The sign "_" in front of the second term and the third term appears since the edge adjacent to vertex 1 has to "move behind" the edge connecting vertex 2 to the only neutral vertex. In the last two terms, the edge adjacent to vertex 1 has to "move behind" the two edges originating from the only neutral vertex. This is why we have pluses in front of these terms. For the precise definition of the operadic compositions in $\mathrm{Br}$, we refer the reader to [5, Sections 7-9].

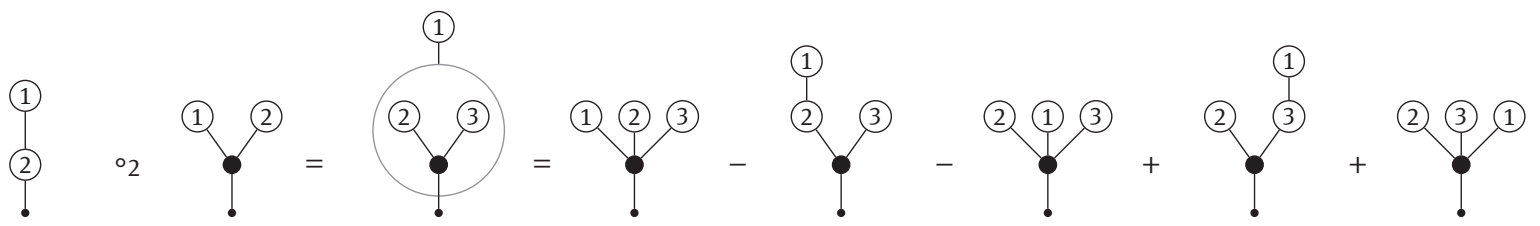

Figure 5. A computation of an elementary insertion.

1 We should remark that the differential $\partial$ defined in [5, equation (8.12)] differs from $\delta$ by the overall sign factor: $\delta=-\partial$. 


\subsection{Remarks on the linear dual of $\mathrm{Br}$}

Let us observe that the linear dual $\operatorname{Br}(n)^{*}$ can be canonically identified with $\operatorname{Br}(n)$ as the vector space. The only difference is that the degree of a brace tree in $\operatorname{Br}(n)^{*}$ equals number of non-root edges $-2 \times$ number of neutral vertices. Using this observation, we will often switch back and forth between various subspaces of $\operatorname{Br}(n)$ (with certain differentials) and their linear duals.

For example, the differential $\delta^{*}$ on the dual complex $\operatorname{Br}(n)^{*}$ is the sum (with appropriate signs)

$$
\delta^{*}(T):=\sum_{e \in \text { Edges. }(T)} \pm \delta_{e}^{*}(T),
$$

where the brace tree $\delta_{e}^{*}(T)$ is obtained from $T$ by contracting the edge $e$ and the set Edges. $(T)$ consists of non-root edges $e$ which satisfy the following property: $e$ either connects two neutral vertices or $e$ is adjacent to one neutral vertex. For instance, for the brace trees shown in Figure 1, we have

$$
\delta^{*}\left(T_{\cup}\right)=T_{1-2}-T_{2-1} .
$$

On the other hand, if $T$ is any brace tree without neutral vertices, then $\delta^{*}(T)=0$.

\section{Computation of the cohomology of $\mathrm{Br}$}

\subsection{The map of collections of dg vector spaces $\Psi:$ Ger $\rightarrow \mathrm{Br}$}

Let us recall (see Appendix A) that the assignment

$$
\mathfrak{j}\left(\left\{a_{1}, a_{2}\right\}\right):=T_{\left\{a_{1}, a_{2}\right\}}
$$

gives us the map of dg operad

$$
\mathrm{j}: \Lambda \text { Lie } \rightarrow \mathrm{Br},
$$

where $\Lambda$ Lie is considered with the zero differential.

We will use $j$ to define a map $\Psi$ of collections

$$
\Psi: \text { Ger } \rightarrow \mathrm{Br} .
$$

For this purpose, we recall [4, Exercise 3.12] that $\operatorname{Ger}(n)$ has the basis formed by the monomials

$$
\left\{a_{i_{11}}, \ldots,\left\{a_{i_{1\left(p_{1}-1\right)}}, a_{i_{1 p_{1}}}\right\} \ldots\right\} \ldots\left\{a_{i_{t 1}}, \ldots,\left\{a_{i_{t\left(p_{t}-1\right)}}, a_{i_{t p_{t}}}\right\} \ldots\right\}
$$

where

$$
\left\{i_{11}, i_{12}, \ldots, i_{1 p_{1}}\right\} \sqcup\left\{i_{21}, i_{22}, \ldots, i_{2 p_{2}}\right\} \sqcup \cdots \sqcup\left\{i_{t 1}, i_{t 2}, \ldots, i_{t p_{t}}\right\}
$$

are ordered partitions of the set $\{1,2, \ldots, n\}$ satisfying the following properties:

- for each $1 \leq \beta \leq t$ the index $i_{\beta p_{\beta}}$ is the biggest among $i_{\beta 1}, \ldots, i_{\beta p_{\beta}}$,

- $i_{1 p_{1}}<i_{2 p_{2}}<\cdots<i_{t p_{t}}$ (in particular, $i_{t p_{t}}=n$ ).

Let $\sigma$ be the permutation in $S_{n}$

$$
\sigma=\left(\begin{array}{cccccccccccccc}
1 & 2 & \ldots & p_{1} & p_{1}+1 & p_{1}+2 & \ldots & p_{1}+p_{2} & \ldots & \ldots & n-p_{t}+1 & n-p_{t}+2 & \ldots & n \\
i_{11} & i_{12} & \ldots & i_{1 p_{1}} & i_{21} & i_{22} & \ldots & i_{2 p_{2}} & \ldots & \ldots & i_{t 1} & i_{t 2} & \ldots & i_{t p_{t}}
\end{array}\right)
$$

corresponding to such a partition (4.4).

Then, for the corresponding monomial (4.3) in the above basis, we set ${ }^{2}$

$$
\begin{aligned}
& \Psi\left(\left\{a_{i_{11}}, \ldots,\left\{a_{i_{1\left(p_{1}-1\right)}}, a_{i_{p_{1}}}\right\} \ldots\right\} \ldots\left\{a_{i_{t 1}}, \ldots,\left\{a_{i_{t\left(p_{t}-1\right)}}, a_{i_{t_{p}}}\right\} \ldots\right\}\right) \\
& \quad=\sigma\left(\Psi\left(\left\{a_{1}, \ldots,\left\{a_{p_{1}-1}, a_{p_{1}}\right\} \ldots\right\}\left\{a_{p_{1}+1}, \ldots,\left\{a_{p_{1}+p_{2}-1}, a_{p_{1}+p_{2}}\right\} \ldots\right\} \ldots\left\{a_{n-p_{t}+1}, \ldots,\left\{a_{n-1}, a_{n}\right\} \ldots\right\}\right)\right), \\
& \Psi\left(\left\{a_{1}, \ldots,\left\{a_{p_{1}-1}, a_{p_{1}}\right\} \ldots\right\}\left\{a_{p_{1}+1}, \ldots,\left\{a_{p_{1}+p_{2}-1}, a_{p_{1}+p_{2}}\right\} \ldots\right\} \ldots\left\{a_{n-p_{t}+1}, \ldots,\left\{a_{n-1}, a_{n}\right\} \ldots\right\}\right) \\
& \quad:=\mu\left(\mathcal{M}_{t} ; j\left(\left\{a_{1}, \ldots,\left\{a_{p_{1}-1}, a_{p_{1}}\right\} \ldots\right\}\right), \mathrm{j}\left(\left\{a_{1}, \ldots,\left\{a_{p_{2}-1}, a_{p_{2}}\right\} \ldots\right\}\right), \ldots, \mathfrak{j}\left(\left\{a_{1}, \ldots,\left\{a_{p_{t}-1}, a_{p_{t}}\right\} \ldots\right\}\right)\right),(4.5)
\end{aligned}
$$

2 Here, we assume that $t \geq 2$. 
where $\mu$ is the operadic multiplication

$$
\operatorname{Br}(t) \otimes\left(\operatorname{Br}\left(p_{1}\right) \otimes \operatorname{Br}\left(p_{2}\right) \otimes \cdots \otimes \operatorname{Br}\left(p_{t}\right)\right) \rightarrow \operatorname{Br}\left(p_{1}+p_{2}+\cdots+p_{t}\right),
$$

and $\mathcal{M}_{t}$ is the vector

$$
\mathcal{M}_{t}:=\underbrace{\left.\left(\ldots\left(T_{a_{1} a_{2} \circ} 0_{1} T_{a_{1} a_{2}}\right) \circ_{1} T_{a_{1} a_{2}}\right) \cdots \circ_{1} T_{a_{1} a_{2}}\right)}_{\circ_{1} \text { appears } t-2 \text { times }} \in \operatorname{Br}(t) .
$$

Finally, if $t=1$, i.e. we deal with a monomial $v \in \Lambda \operatorname{Lie}(n)$, then we set

$$
\Psi(v):=\mathfrak{j}(v) .
$$

For example, the vector $\mathcal{M}_{3}=T_{a_{1} a_{2}{ }^{\circ}} T_{a_{1} a_{2}}$ is shown in Figure 6 and the vector $\Psi\left(a_{1} a_{2}\left\{a_{3}, a_{4}\right\}\right) \in \operatorname{Br}(4)$ is shown in Figure 7.

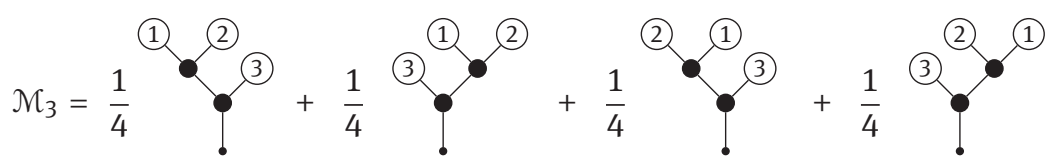

Figure 6. The vector $\mathcal{M}_{3} \in \operatorname{Br}(3)$.

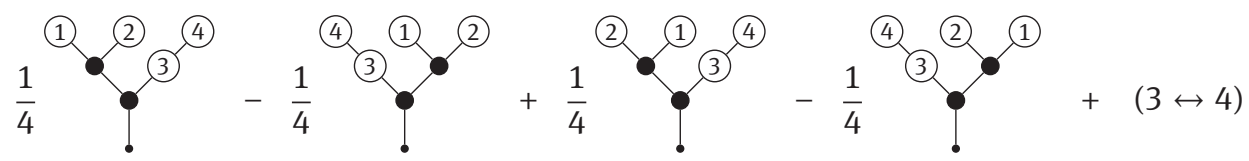

Figure 7. The vector $\Psi\left(a_{1} a_{2}\left\{a_{3}, a_{4}\right\}\right) \in \operatorname{Br}(4)$.

We claim that

Proposition 4.1. Equations (4.5) and (4.7) define a map of collections of dg vector spaces

$$
\Psi: \text { Ger } \rightarrow \text { Br. }
$$

Furthermore, the induced map

$$
H^{\bullet}(\Psi): \text { Ger } \rightarrow H^{\bullet}(\mathrm{Br})
$$

is compatible with the operadic multiplications.

Proof. The first statement follows from the fact that the vectors $T_{a_{1} a_{2}}, T_{\left\{a_{1}, a_{2}\right\}} \in \operatorname{Br}(2)$ are $\delta$-cocycles. The second statement follows from Claim A.1 proved in Appendix A.

\subsection{The refinement of Theorem 1.1}

We will prove the following refined version of Theorem 1.1:

Theorem 4.2. The map of dg collections $\Psi$ defined above induces an isomorphism of graded operads

$$
H^{\bullet}(\mathrm{Br}) \cong \mathrm{Ger} .
$$

We will prove that $\Psi$ induces an isomorphism $H^{*}(\operatorname{Br}(n))=\operatorname{Ger}(n)$ by induction on $n$.

For $n=1$ there is nothing to show. So suppose we know that $H^{\bullet}(\operatorname{Br}(j))=\operatorname{Ger}(j)$ for $j=1,2 \ldots, n-1$ and let us tackle the statement for $j=n$. As outlined in the introduction, we split

$$
\operatorname{Br}(n)=V_{0}(n) \stackrel{\delta_{0}}{\delta_{1}} \begin{gathered}
\delta_{0} \\
\oplus V_{0}(n),
\end{gathered}
$$


where $V_{\bullet}(n)$ is the subspace of $\operatorname{Br}(n)$ spanned by brace trees whose lowest non-root vertex is neutral, while $V_{\mathrm{o}}(n)$ is the subspace of $\operatorname{Br}(n)$ spanned by brace trees whose lowest non-root vertex is labeled. Again as mentioned before, we then find that

$$
H^{\bullet}(\operatorname{Br}(n))=\left(\operatorname{ker} H^{\bullet}\left(\delta_{1}\right)\right) \oplus\left(\operatorname{coker} H^{\bullet}\left(\delta_{1}\right)\right),
$$

where

$$
H^{\bullet}\left(\delta_{1}\right): H^{\bullet}\left(V_{\circ}(n), \delta_{0}\right) \rightarrow H^{\bullet}\left(V_{\bullet}(n), \delta_{0}\right) .
$$

is the induced map on $\delta_{0}$-cohomologies.

\subsection{Computing $H^{\bullet}\left(V_{\circ}(n), \delta_{0}\right)$}

Following remarks in Section 3.1, we begin by computing the graded operad $H^{*}\left(V_{\circ}^{*}(n), \delta_{0}^{*}\right)$ for the dual of the complex $\left(V_{\mathrm{o}}(n), \delta_{0}\right)$ :

Claim 4.3. We claim that

$$
H^{\bullet}\left(V_{\circ}^{*}(n), \delta_{0}^{*}\right) \cong \mathbf{s}^{n-1} \mathbb{K}^{n !} \cong \mathbf{s}^{n-1} \mathbb{K}\left[S_{n}\right]
$$

as $S_{n}$-modules. Moreover, the class corresponding to a permutation $\lambda \in S_{n}$ is represented by the brace tree $T_{\lambda}^{n}$ shown in Figure 12.

Proof. We proceed by induction on $n$. For $n=1$ the statement is clear. Otherwise split:

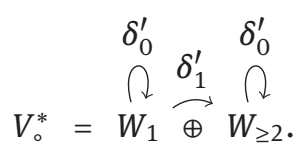

Here $W_{1}$ is spanned by brace trees in which the lowest non-root vertex has exactly one child and $W_{\geq 2}$ is spanned by brace trees in which the lowest non-root vertex has at least two children. It is easy to see that $\delta_{1}^{\prime}$ is surjective and that its kernel is spanned by brace trees whose lowest non-root vertex has a labeled vertex as a child. The complex $\left(\operatorname{ker} \delta_{1}^{\prime}, \delta_{0}^{\prime}\right)$ is isomorphic to $\left(V_{\circ}^{*}(n-1), \delta_{0}^{*}\right)$.

Thus the induction hypothesis implies that $H^{*}\left(V_{0}^{*}(n), \delta_{0}^{*}\right) \cong \mathbf{s}^{n-1} \mathbb{K}\left[S_{n}\right]$ as graded vector spaces. The compatibility of the resulting isomorphism with the $S_{n}$-action is obvious.

Remark 4.4. Recall that every brace tree $T \in \operatorname{Br}(n)^{*}$ without neutral vertices is automatically $\delta^{*}$-closed and hence $\delta_{0}^{*}$-closed. Therefore, by Claim 4.3, for every brace tree $T \in \operatorname{Br}(n)^{*}$ without neutral vertices, there exists a vector $T^{\prime} \in V_{\circ}^{*}(n)$ such that $T-\delta_{0}^{*} T^{\prime}$ is a linear combination of string-like brace trees, i.e. brace trees of the form $T_{\lambda}^{n}$ (see Figure 12).

\subsection{Computing $H^{\bullet}\left(V_{.}(n), \delta_{0}\right)$}

To compute $H^{\bullet}\left(V_{\bullet}(n), \delta_{0}\right)$, we filter the cochain complex $\left(V_{\bullet}(n), \delta_{0}\right)$ by the number of children of the lowest non-root vertex:

$$
\mathbf{0}=\mathcal{F}^{1} V_{\bullet}(n) \subset \mathcal{F}^{2} V \cdot(n) \subset \mathcal{F}^{3} V_{\bullet}(n) \subset \cdots \subset \mathcal{F}^{n} V_{\bullet}(n)=V_{\bullet}(n) .
$$

Here $\mathcal{F}^{p} V$. $(n)$ is spanned by brace trees whose lowest non-root vertex has $\leq p$ children. Then we consider the spectral sequence associated to this filtration.

The first differential, say $d_{0}$, splits vertices except for the lowest non-root vertex. Hence,

$$
\operatorname{Gr} V .(n) \cong(\mathbf{s} \Lambda \operatorname{coAs} 。 \odot \mathrm{Br})(n),
$$

where $\mathbf{s} \Lambda$ coAs。 is the collection with

$$
\mathbf{s} \Lambda \operatorname{coAs}_{\circ}(q)= \begin{cases}\mathbf{s}^{2-q} \mathbb{K}\left[S_{q}\right] \otimes \operatorname{sgn}_{q} & \text { if } q \geq 2, \\ \mathbf{0} & \text { otherwise }\end{cases}
$$




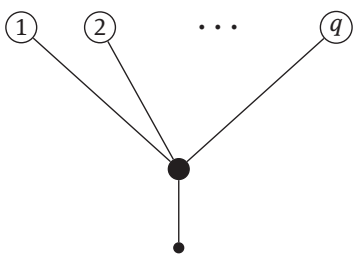

Figure 8. The brace tree $T_{q}^{*}$.

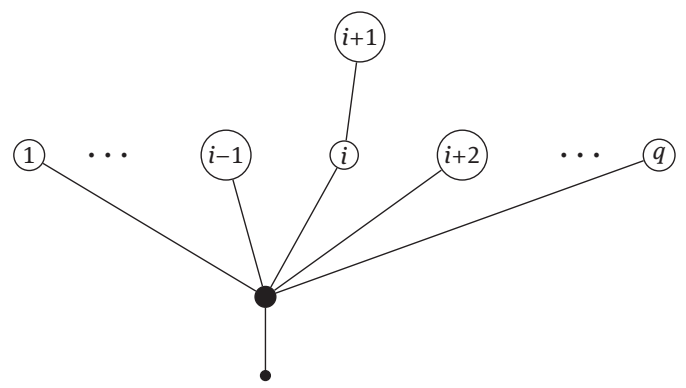

Figure 9. The brace tree $T_{q, i}^{*}$

Therefore, by inductive hypothesis, we conclude that

$$
E_{1} V_{\bullet}(n):=H^{\bullet}\left(\operatorname{Gr} V \cdot(n), d_{0}\right) \cong(\mathbf{s} \Lambda \operatorname{coAs} 。 \odot \operatorname{Ger})(n) .
$$

Moreover, the cohomology class in $H^{\bullet}\left(\mathrm{Gr}^{q} V \cdot(n), d_{0}\right)$ corresponding to the vector

$$
\mathbf{s}^{2-q} \mathrm{id}_{q} \otimes\left(v_{1} \otimes \cdots \otimes v_{q}\right) \in \mathbf{s} \Lambda \operatorname{coAs} 。(q) \otimes\left(\operatorname{Ger}\left(n_{1}\right) \otimes \cdots \otimes \operatorname{Ger}\left(n_{q}\right)\right)
$$

is represented by the $d_{0}$-cocycle

$$
\mu\left(T_{q}^{*} ; \Psi\left(v_{1}\right), \Psi\left(v_{2}\right), \ldots, \Psi\left(v_{q}\right)\right) \in \operatorname{Br}(n),
$$

where $\mu$ is the operadic multiplication on $\mathrm{Br}, n=n_{1}+\cdots+n_{q}, T_{q}^{*}$ is the brace tree shown in Figure 8 , and $\Psi$ is the map of collections (4.2).

Before proceeding to further pages of this spectral sequence, we need to fix some conventions ${ }^{3}$. First, we denote by $\mathcal{A}_{r}^{q}(r \geq 0)$ the following subspaces of $\mathcal{F}^{q} V_{\bullet}(n)$ :

$$
\mathcal{A}_{r}^{q}:=\left\{v \in \mathcal{F}^{q} V_{\bullet}(n): \delta_{0}(v) \in \mathcal{F}^{q-r} V_{.}(n)\right\} .
$$

For example, $\mathcal{A}_{0}^{q}=\mathcal{F}^{q} V$. $(n)$ and vectors in $\mathcal{A}_{1}^{q}$ represent cocycles in $\mathrm{Gr}^{q} V$. $(n)$. By the construction of the spectral sequence [25, Construction 5.4.6], the components of the $r$-th page are the quotients

$$
E_{r}^{q}:=\frac{\mathcal{A}_{r}^{q}}{\delta_{0}\left(\mathcal{A}_{r-1}^{q+r-1}\right)+\mathcal{A}_{r-1}^{q-1}} .
$$

The results of the computation of $E_{2} V_{\bullet}(n):=H^{\bullet}\left(E_{1} V_{\bullet}(n), d_{1}\right)$ are listed in the following claim:

Claim 4.5. For $E_{2} V_{\bullet}(n):=H^{\bullet}\left(E_{1} V_{\bullet}(n), d_{1}\right)$, we have

$$
E_{2} V .(n) \cong \operatorname{Com} \odot \Lambda \operatorname{Lie}(n) / \Lambda \operatorname{Lie}(n) \oplus \mathbf{S}(\Lambda \operatorname{Com} \odot \Lambda \operatorname{Lie}(n) / \Lambda \operatorname{Lie}(n)) .
$$

More precisely,

$$
E_{2}^{q} V_{\bullet}(n) \cong \begin{cases}\operatorname{Com} \odot \Lambda \operatorname{Lie}(n) / \Lambda \operatorname{Lie}(n) \oplus \bigoplus_{n_{1}+n_{2}=n} \operatorname{Ind}_{S_{n_{1} \times S_{n_{2}}} S_{n}}\left(\operatorname{sgn}_{2} \otimes_{S_{2}}\left(\Lambda \operatorname{Lie}\left(n_{1}\right) \otimes \Lambda \operatorname{Lie}\left(n_{2}\right)\right)\right) & \text { if } q=2, \\ \bigoplus_{n_{1}+\cdots+n_{q}=n} \operatorname{Ind}_{S_{n_{1}} \times \cdots \times S_{n_{q}}}^{S_{n}}\left(\mathbf{s}^{2-q} \operatorname{sgn}_{q} \otimes_{S_{q}}\left(\Lambda \operatorname{Lie}\left(n_{1}\right) \otimes \cdots \otimes \Lambda \operatorname{Lie}\left(n_{q}\right)\right)\right) & \text { if } 3 \leq q \leq n, \\ 0 & \text { otherwise. }\end{cases}
$$

The classes corresponding to vectors in $\operatorname{Com} \odot \Lambda \mathrm{Lie}(n) / \Lambda \mathrm{Lie}(n)$ are represented in $\mathcal{A}_{2}^{2}$ by cocycles $($ in $(\operatorname{Br}(n), \delta))$ which are obtained by applying $\Psi$ (see (4.2)) to linear combinations of monomials (4.3) in $\operatorname{Ger}(n)$ with $t \geq 2$.

3 We use the cohomological version of the notational conventions from [25, Construction 5.4.6]. 
If $q \geq 3$, the class corresponding to the vector

$$
\mathbf{s}^{2-q} 1_{q} \otimes\left(v_{1} \otimes \cdots \otimes v_{q}\right) \in \mathbf{s} \Lambda \operatorname{Com} \odot \Lambda \operatorname{Lie}(n)
$$

is represented in $\mathcal{A}_{2}^{q}$ by the cochain

$$
\begin{aligned}
u_{q}:=\frac{1}{q !} \sum_{\sigma \in S_{q}}(-1)^{|\sigma|} \mu\left(\sigma\left(T_{q}^{*}\right) \otimes \mathfrak{j}\left(v_{1}\right) \otimes \mathfrak{j}\left(v_{2}\right) \otimes \cdots \otimes \mathfrak{j}\left(v_{q}\right)\right) \\
\quad+\frac{1}{q !} \sum_{i=1}^{q-1} \sum_{\substack{\sigma \in S_{q} \\
\sigma(i)<\sigma(i+1)}}(-1)^{|\sigma|} \mu\left(\sigma\left(T_{q, i}^{*}\right) \otimes \mathfrak{j}\left(v_{1}\right) \otimes \mathfrak{j}\left(v_{2}\right) \otimes \cdots \otimes \mathfrak{j}\left(v_{q}\right)\right),
\end{aligned}
$$

where $\mu$ is the operadic composition

$$
\mu: \operatorname{Br}(q) \otimes \operatorname{Br}\left(m_{1}\right) \otimes \cdots \otimes \operatorname{Br}\left(m_{q}\right) \rightarrow \operatorname{Br}\left(m_{1}+\cdots+m_{q}\right),
$$

$\mathrm{j}$ is the operad map in (1.2), and $T_{q}^{*}\left(\right.$ resp. $\left.T_{q, i}^{*}\right)$ is the brace tree shown in Figure 8 (resp. Figure 9). Finally, the class corresponding to the vector

$$
1_{2} \otimes\left(v_{1} \otimes v_{2}\right) \in \operatorname{sgn}_{2} \otimes_{S_{2}}\left(\Lambda \operatorname{Lie}\left(n_{1}\right) \otimes \Lambda \operatorname{Lie}\left(n_{2}\right)\right)
$$

is represented in $\mathcal{A}_{2}^{2}$ by the cochain

$$
\frac{1}{2} \mu\left(\left(T_{\cup}-T_{\cup}^{\mathrm{opp}}\right) \otimes \mathrm{j}\left(v_{1}\right) \otimes \mathfrak{j}\left(v_{2}\right)\right),
$$

where $T_{\cup}$ and $T_{\cup}^{\mathrm{opp}}$ are shown in Figure 1.

Remark 4.6. Note that the vector (4.22) is not closed in $\left(V .(n), \delta_{0}\right)$. It is merely a representative of an element in $E_{2}^{q}$, i.e. a vector $v \in \mathcal{F}^{q} V$. $(n)$ such that $\delta_{0}(v) \in \mathcal{F}^{q-2} V$. $(n)$.

Proof. The differential $d_{1}$ on $E_{1} V .(n)$ splits the lowest non-root vertex producing a neutral child node with two children. To describe this cochain complex, we consider the free Gerstenhaber algebra $\mathrm{Ger}_{n}$ in $n$ auxiliary variables $a_{1}, a_{2}, \ldots, a_{n}$ of degree zero. Forgetting the bracket $\{\cdot, \cdot\}$ on $\operatorname{Ger}_{n}$ we can view it merely as the free commutative algebra (without unit)

$$
\operatorname{Ger}_{n}=\operatorname{Com}\left(\Lambda \operatorname{Lie}_{n}\right)
$$

generated by the free $\Lambda$ Lie-algebra $\Lambda \mathrm{Lie}_{n}$ in the auxiliary variables $a_{1}, a_{2}, \ldots, a_{n}$

Next, we introduce the cofree coassociative coalgebra

$$
\operatorname{coAs}\left(\mathbf{s}^{-1} \operatorname{Ger}_{n}\right)=\bigoplus_{q \geq 1}\left(\mathbf{s}^{-1} \operatorname{Ger}_{n}\right)^{\otimes q}
$$

and equip it with the coderivation $\mathfrak{d}$ defined by the equation ${ }^{4}$

$$
p \circ \mathfrak{d}\left(\mathbf{s}^{-1} v_{1} \otimes \cdots \otimes \mathbf{s}^{-1} v_{q}\right)= \begin{cases}(-1)^{\left|v_{1}\right|+1} \mathbf{s}^{-1} v_{1} v_{2} & \text { if } q=2, \\ 0 & \text { otherwise, }\end{cases}
$$

where $v_{i} \in \operatorname{Ger}_{n}$ and $p$ is the canonical projection

$$
p: \operatorname{coAs}\left(\mathbf{s}^{-1} \mathrm{Ger}_{n}\right) \rightarrow \mathbf{s}^{-1} \operatorname{Ger}_{n} .
$$

It is easy to see that the coderivation $\mathfrak{d}$ has degree 1 . Moreover, due to associativity of the multiplication on $\mathrm{Ger}_{n}$, we have

$$
\mathfrak{d}^{2}=0 .
$$

In other words, $\mathfrak{d}$ is a differential on the coalgebra (4.24).

4 Note that, since the coalgebra (4.24) is cofree, any coderivation $\mathfrak{d}$ is uniquely determined by its composition with the projection (4.26). 
For our purposes we need the following truncation of the cochain complex $\mathbf{s}^{2} \operatorname{coAs}\left(\mathbf{s}^{-1} \mathrm{Ger}_{n}\right)$ :

$$
\mathbf{s}^{2} T^{\prime}\left(\mathbf{s}^{-1} \operatorname{Ger}_{n}\right)=\bigoplus_{q \geq 2} \mathbf{s}^{2}\left(\mathbf{s}^{-1} \operatorname{Ger}_{n}\right)^{\otimes q}
$$

with the differential $\mathfrak{d}^{\prime}$ given by the formula

$$
\mathfrak{d}^{\prime}\left(\mathbf{s}^{2}\left(\mathbf{s}^{-1} v_{1} \otimes \mathbf{s}^{-1} v_{2} \otimes \cdots \otimes \mathbf{s}^{-1} v_{q}\right)\right)=\left\{\begin{array}{ll}
\mathbf{s}^{2} \mathfrak{d}\left(\mathbf{s}^{-1} v_{1} \otimes \mathbf{s}^{-1} v_{2} \otimes \cdots \otimes \mathbf{s}^{-1} v_{q}\right) & \text { if } q>2, \\
0 & \text { if } q=2,
\end{array} v_{i} \in \operatorname{Ger}_{n} .\right.
$$

It is not hard to see that $E_{1} V_{\text {. }}(n)$ (4.16) is isomorphic to the subspace of $\mathbf{s}^{2} T^{\prime}\left(\mathbf{s}^{-1} \mathrm{Ger}_{n}\right)$ which is spanned by tensor monomials

$$
\mathbf{s}^{2}\left(\mathbf{s}^{-1} v_{1} \otimes \mathbf{s}^{-1} v_{2} \otimes \cdots \otimes \mathbf{s}^{-1} v_{q}\right), \quad v_{i} \in \operatorname{Ger}_{n}, \quad 2 \leq q \leq n
$$

in which each variable from the set $\left\{a_{1}, a_{2}, \ldots, a_{n}\right\}$ appears exactly once. It is easy to see that this subspace is a subcomplex with respect to $\mathfrak{d}^{\prime}$ and, moreover, the differential $d_{1}$ coincides with the restriction of $\mathfrak{d}^{\prime}$ up to a total sign.

Since the augmentation

$$
\cdots \stackrel{\mathfrak{d}}{\rightarrow}\left(\mathbf{s}^{-1} \mathrm{Ger}_{n}\right)^{\otimes 2} \stackrel{\mathfrak{d}}{\rightarrow} \mathbf{s}^{-1} \mathrm{Ger}_{n} \stackrel{0}{\rightarrow} \mathbb{K}
$$

of the cochain complex (4.24) computes the Hochschild homology

$$
H H_{-\bullet}\left(S\left(\Lambda \operatorname{Lie}_{n}\right), \mathbb{K}\right)
$$

of the free commutative algebra $S\left(\Lambda \mathrm{Lie}_{n}\right)$ (with unit) with the trivial coefficients, we conclude that ${ }^{5}[17$, Section 3.2]

$$
H^{\bullet}\left(\operatorname{coAs}\left(\mathbf{s}^{-1} \operatorname{Ger}_{n}\right), \mathfrak{d}\right)=\bigoplus_{q \geq 1} S^{q}\left(\mathbf{s}^{-1} \Lambda \operatorname{Lie}_{n}\right)
$$

and the cohomology class of the symmetric word $\left(\mathbf{s}^{-1} v_{1}, \mathbf{s}^{-1} v_{2}, \ldots, \mathbf{s}^{-1} v_{q}\right) \in S^{q}\left(\mathbf{s}^{-1} \Lambda\right.$ Lie $\left._{n}\right)$ is represented by the cocycle

$$
\frac{1}{q !} \sum_{\sigma \in S_{q}}(-1)^{\varepsilon\left(\sigma, v_{1}, \ldots, v_{q}\right)}\left(\mathbf{s}^{-1} v_{\sigma(1)}, \mathbf{s}^{-1} v_{\sigma(2)}, \ldots, \mathbf{s}^{-1} v_{\sigma(q)}\right) \in\left(\mathbf{s}^{-1} \mathrm{Ger}_{n}\right)^{\otimes q},
$$

where the sign factors $(-1)^{\varepsilon\left(\sigma, v_{1}, \ldots, v_{q}\right)}$ are determined by the Koszul rule. When we pass to the truncation (4.27) of the Hochschild complex, the cohomology in the terms $\left(\mathbf{s}^{-1} \mathrm{Ger}_{n}\right)^{\otimes} q$ for $q \geq 3$ does not change.

As for $q=2$, all vectors in $\left(\mathbf{s}^{-1} \mathrm{Ger}_{n}\right)^{\otimes 2}$ are cocycles in the truncated complex (4.27).

Since for every pair of vectors $v_{1}, v_{2} \in \mathrm{Ger}_{n}$,

$$
\begin{aligned}
& \mathbf{s}^{-1} v_{1} \otimes \mathbf{s}^{-1} v_{2}=\frac{(-1)^{\left|v_{1}\right|}}{2} \mathbf{s}^{-1} \otimes \mathbf{s}^{-1}\left(v_{1} \otimes v_{2}+(-1)^{\left|v_{1}\right|\left|v_{2}\right|} v_{2} \otimes v_{1}\right) \\
&+\frac{1}{2}\left(\mathbf{s}^{-1} v_{1} \otimes \mathbf{s}^{-1} v_{2}+(-1)^{\left(\left|v_{1}\right|+1\right)\left(\left|v_{2}\right|+1\right)} \mathbf{s}^{-1} v_{2} \otimes \mathbf{s}^{-1} v_{1}\right),
\end{aligned}
$$

we have the obvious decomposition

$$
\left(\mathbf{s}^{-1} \operatorname{Ger}_{n}\right)^{\otimes 2} \cong \mathbf{s}^{-2} S^{\geq 2}\left(\Lambda \operatorname{Lie}_{n}\right) \oplus S^{2}\left(\mathbf{s}^{-1} \operatorname{Ger}_{n}\right),
$$

where $S^{2}\left(\mathbf{s}^{-1} \mathrm{Ger}_{n}\right)$ is precisely the kernel of

$$
\left(\mathbf{s}^{-1} \mathrm{Ger}_{n}\right)^{\otimes 2} \stackrel{\mathfrak{d}}{\rightarrow} \mathbf{s}^{-1} \mathrm{Ger}_{n}
$$

and $\mathbf{s}^{-2} S^{\geq 2}\left(\Lambda \mathrm{Lie}_{n}\right)$ is (up to the degree shift) the image of (4.32).

5 In [17], J.-L. Loday only considers the case when the symmetric algebra is generated by an "ungraded" vector space and $H H$. is computed with coefficients in the symmetric algebra. However, the obvious generalization to the Koszul resolution to the graded case can be applied in the straightforward manner in our case. 
Combining this observation with the knowledge about homology (4.30), we conclude that

$$
H^{\bullet}\left(\mathbf{s}^{2} T^{\prime}\left(\mathbf{s}^{-1} \operatorname{Ger}_{n}\right), \mathfrak{d}^{\prime}\right) \cong S^{\geq 2}\left(\Lambda \mathrm{Lie}_{n}\right) \oplus \bigoplus_{q \geq 2} \mathbf{s}^{2} S^{q}\left(\mathbf{s}^{-1} \Lambda \mathrm{Lie}_{n}\right) .
$$

On the other hand, $E_{1} V .(n)$ is isomorphic to the direct summand of the cochain complex $\left(\mathbf{s}^{2} T^{\prime}\left(\mathbf{s}^{-1} \mathrm{Ger}_{n}\right), \mathfrak{d}^{\prime}\right)$.

Thus the first two statements of Claim 4.5 follow from (4.33). To deduce the remaining statements, we use the description of cohomology classes in $H^{*}\left(\mathrm{Gr} V .(n), d_{0}\right)$ corresponding to vectors in $(\mathbf{s} \Lambda$ coAs。 $\odot$ Ger $)(n)$ (see equation (4.17)).

The most involving statement is about the class corresponding to the vector

$$
\mathbf{s}^{2-q_{1}} 1_{q} \otimes\left(v_{1} \otimes \cdots \otimes v_{q}\right) \in \mathbf{s} \Lambda \operatorname{Com} \odot \Lambda \operatorname{Lie}(n)
$$

for $q \geq 3$. Using the information about the $E_{1}$ page, we know that (4.34) is represented in $\mathcal{A}_{1}^{q}$ by the vector

$$
f_{q}:=\frac{1}{q !} \sum_{\sigma \in S_{q}}(-1)^{|\sigma|} \mu\left(\sigma\left(T_{q}^{*}\right) \otimes \mathfrak{j}\left(v_{1}\right) \otimes \mathfrak{j}\left(v_{2}\right) \otimes \cdots \otimes \mathfrak{j}\left(v_{q}\right)\right) .
$$

A direct computation shows that

$$
\delta_{0}\left(\sum_{\sigma \in S_{q}}(-1)^{|\sigma|} \sigma\left(T_{q}^{*}\right)\right)+\delta_{0}\left(\sum_{i=1}^{q-1} \sum_{\substack{\sigma \in S_{q} \\ \sigma(i)<\sigma(i+1)}}(-1)^{|\sigma|} \sigma\left(T_{q, i}^{*}\right)\right) \in \mathcal{F}^{q-2} V \cdot(n) .
$$

Therefore the sum

$$
u_{q}=f_{q}+\frac{1}{q !} \sum_{i=1}^{q-1} \sum_{\substack{\sigma \in S_{q} \\ \sigma(i)<\sigma(i+1)}}(-1)^{|\sigma|} \mu\left(\sigma\left(T_{q, i}^{*}\right) \otimes \mathfrak{j}\left(v_{1}\right) \otimes \mathfrak{j}\left(v_{2}\right) \otimes \cdots \otimes \mathfrak{j}\left(v_{q}\right)\right)
$$

belongs to $\mathcal{A}_{2}^{q}$ and represents the element in $E_{2}^{q}$ corresponding to (4.34). Claim 4.5 is proved.

Due to Lemma B.4 from Appendix B, this spectral sequence degenerates at the second page, i.e.

$$
E_{\infty} V \cdot(n)=E_{2} V \cdot(n) .
$$

Hence Claim 4.5 implies the following statement.

Claim 4.7. For the complex $\left(V .(n), \delta_{0}\right)$ we have

$$
H^{\bullet}\left(V .(n), \delta_{0}\right) \cong \operatorname{Com} \odot \Lambda \operatorname{Lie}(n) / \Lambda \operatorname{Lie}(n) \oplus \mathbf{s}(\Lambda \operatorname{Com} \odot \Lambda \operatorname{Lie}(n) / \Lambda \operatorname{Lie}(n)) .
$$

Cohomology classes in $\left(V_{\bullet}(n), \delta_{0}\right)$ corresponding to vectors in $\operatorname{Com} \odot \Lambda \mathrm{Lie}(n) / \Lambda \mathrm{Lie}(n)$ are represented by cocycles (in $(\mathrm{Br}(n), \delta)$ ) which are obtained by applying $\Psi$ (4.2) to linear combinations of monomials (4.3) in $\mathrm{Ger}(n)$ with $t \geq 2$. The class corresponding to the vector

$$
\mathbf{s}^{2-q} 1_{q} \otimes\left(v_{1} \otimes \cdots \otimes v_{q}\right) \in \mathbf{s} \Lambda \operatorname{Com} \odot \Lambda \operatorname{Lie}(n), \quad q \geq 2
$$

is represented in $\left(V \cdot(n), \delta_{0}\right)$ by the $\delta_{0}$-cocycle of the form

$$
u_{q}+\cdots
$$

where $u_{q}$ is the vector given in (4.22) and $\cdots$ denotes the sum of terms in $\mathcal{F}^{q-1} V \cdot(n)$.

Remark 4.8. One may, of course, dualize the statement of Claim 4.7. The dual statement says that

$$
H^{\bullet}\left(V_{\bullet}^{*}(n), \delta_{0}^{*}\right) \cong X^{*} \oplus U^{*},
$$

where $X^{*} \subset \operatorname{Ger}(n)^{*}$ is the kernel of $\operatorname{Ger}(n)^{*} \rightarrow \Lambda \operatorname{Lie}(n)^{*}$ and $U^{*}$ is the linear dual of

$$
\mathbf{s}(\Lambda \operatorname{Com} \odot \Lambda \operatorname{Lie}(n) / \Lambda \operatorname{Lie}(n)) \text {. }
$$




\subsection{A technical claim about $H^{\bullet}\left(\delta_{1}^{*}\right): H^{\bullet}\left(V_{\bullet}(n)^{*}, \delta_{0}^{*}\right) \rightarrow H^{\bullet}\left(V_{\circ}(n)^{*}, \delta_{0}^{*}\right)$}

Let summarize what we proved so far.

- $\quad$ First, due to Claim 4.3,

$$
H^{k}\left(V_{\circ}(n), \delta_{0}\right)= \begin{cases}\mathbb{K}\left[S_{n}\right] & \text { if } k=1-n, \\ 0 & \text { otherwise. }\end{cases}
$$

- $\quad$ Second, due to Claim 4.7,

$$
H^{\bullet}\left(V \cdot(n), \delta_{0}\right) \cong \operatorname{Com} \odot \Lambda \operatorname{Lie}(n) / \Lambda \operatorname{Lie}(n) \oplus \mathbf{s}(\Lambda \operatorname{Com} \odot \Lambda \operatorname{Lie}(n) / \Lambda \operatorname{Lie}(n))
$$

- The subspace

$$
\mathbf{s}(\Lambda \operatorname{Com} \odot \Lambda \operatorname{Lie}(n) / \Lambda \operatorname{Lie}(n))
$$

is concentrated in the degree $2-n$, and the subspace Com $\odot \Lambda \operatorname{Lie}(n) / \Lambda \operatorname{Lie}(n)$ lives in degrees $2-n \leq \bullet \leq 0$. Thus the operator $H^{\bullet}\left(\delta_{1}\right)$ sends vectors of $H^{1-n}\left(V_{\circ}(n), \delta_{0}\right)$ to the space $H^{2-n}\left(V_{\bullet}(n), \delta_{0}\right)$. Hence,

$$
H^{k}(\operatorname{Br}(n)) \cong \begin{cases}H^{1-n}\left(V_{\circ}(n), \delta_{0}\right) \cap \operatorname{ker}\left(H^{\bullet}\left(\delta_{1}\right)\right) & \text { if } k=1-n, \\ H^{2-n}\left(V_{\bullet}(n), \delta_{0}\right) / \operatorname{Im}\left(H^{\bullet}\left(\delta_{1}\right)\right) & \text { if } k=2-n, \\ H^{k}\left(V_{\bullet}(n), \delta_{0}\right) & \text { if } 3-n \leq k \leq 0, \\ 0 & \text { otherwise. }\end{cases}
$$

Let us prove the following.

Claim 4.9. The map

$$
H^{\bullet}(\mathrm{j}): \Lambda \operatorname{Lie}(n) \rightarrow H^{1-n}(\operatorname{Br}(n))
$$

is injective. In particular,

$$
\operatorname{dim} H^{1-n}(\operatorname{Br}(n)) \geq(n-1) !
$$

Proof. Since $\operatorname{Br}(n)$ lives is degrees $1-n \leq \bullet \leq 0$,

$$
H^{1-n}(\operatorname{Br}(n))=\operatorname{Br}(n)^{1-n} \cap \operatorname{ker}(\delta) .
$$

It is not hard to prove (by induction on $n$ ) that

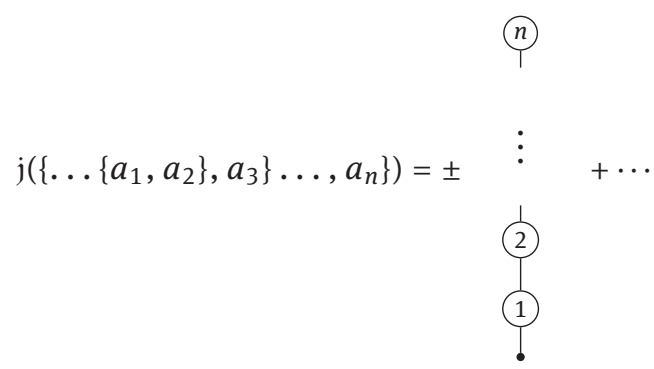

where $\cdots$ is the sum of braces trees which do not involve string-like brace trees with vertex 1 at the lowest position. Therefore, for every permutation $\tau \in S_{\{2,3, \ldots, n\}}$, we have

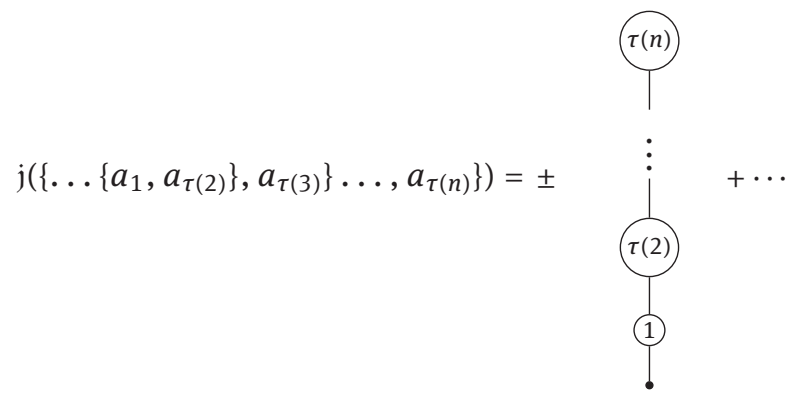




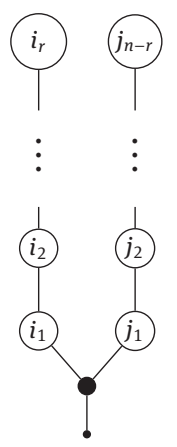

Figure 10. The brace tree $T_{\|, \sigma, r}$.

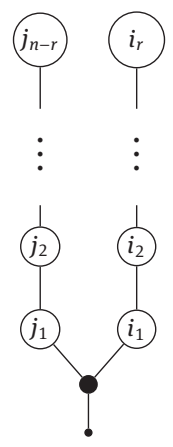

Figure 11. The brace tree $T_{\|, \sigma, r}^{\mathrm{opp}}$.

where, as above, $\cdots$ is the sum of braces trees which do not involve string-like brace trees with vertex 1 at the lowest position. Thus, $\mathfrak{j}$ gives us $(n-1)$ ! linearly independent vectors

$$
\left.\left\{\mathfrak{j}\left(\left\{\ldots\left\{a_{1}, a_{\tau(2)}\right\}, a_{\tau(3)}\right\} \ldots, a_{\tau(n)}\right\}\right)\right\}_{\tau \in S_{\{2,3, \ldots, n\}}}
$$

in (4.43). Since the set

$$
\left.\left\{\left\{\ldots\left\{a_{1}, a_{\tau(2)}\right\}, a_{\tau(3)}\right\} \ldots, a_{\tau(n)}\right\}\right\}_{\tau \in S_{\{2,3, \ldots, n\}}}
$$

is a basis of $\Lambda \operatorname{Lie}(n)$, the claim follows.

To prove the other inequality

$$
\operatorname{dim} H^{1-n}(\operatorname{Br}(n)) \leq(n-1) !
$$

we need the following technical statement:

Claim 4.10. Let $1 \leq r \leq n-1$ and

$$
\sigma=\left(\begin{array}{cccccccc}
1 & 2 & \ldots & r & r+1 & r+2 & \ldots & n \\
i_{1} & i_{2} & \ldots & i_{r} & j_{1} & j_{2} & \ldots & j_{n-r}
\end{array}\right)
$$

be a permutation in $S_{n}$. Let $T_{\|, \sigma, r}\left(\right.$ resp. $T_{\|, \sigma, r}^{\mathrm{opp}}$ ) be the brace tree shown in Figure 10 (resp. in Figure 11).

The vector

$$
\frac{1}{2}\left(T_{\|, \sigma, r}+(-1)^{r(n-r)} T_{\|, \sigma, r}^{\text {opp }}\right)
$$

is a cocycle in the dual complex $\left(V \cdot(n)^{*}, \delta_{0}^{*}\right)$ representing a cohomology class corresponding to a vector in $U^{*}$, i.e. the dual of the subspace (4.41).

Moreover, the vector

$$
\frac{1}{2} \delta_{1}^{*}\left(T_{\|, \sigma, r}+(-1)^{r(n-r)} T_{\|, \sigma, r}^{\text {opp }}\right)
$$

is cohomologous in $\left(V_{\circ}^{*}(n), \delta_{0}^{*}\right)$ to

$$
\sum_{\tau \in \mathrm{Sh}_{r, n-r}}(-1)^{|\tau|} T_{\sigma \circ \tau^{-1}}^{n},
$$

where $(-1)^{|\tau|}$ is the sign of the permutation $\tau$ and $\left\{T_{\lambda}^{n}\right\}_{\lambda \in S_{n}}$ be the family of brace trees shown in Figure 12.

Proof. First, every brace tree with exactly one neutral vertex (at the lowest position) is a cocycle in the complex $\left(V_{\cdot}(n)^{*}, \delta_{0}^{*}\right)$.

To prove that the vector (4.45) belongs to $U^{*}$, we need to show that the pairing

$$
\left(T_{\|, \sigma, r}+(-1)^{r(n-r)} T_{\|, \sigma, r}^{\text {opp }}\right)(w)=0,
$$

where $w$ is a cocycle representing a cohomology class in the graded operad $H^{\bullet}\left(V \cdot(n), \delta_{0}\right)$ corresponding a vector in $\operatorname{Com} \odot \Lambda \operatorname{Lie}(n) / \Lambda \operatorname{Lie}(n)$.

Due to Claim 4.7, we may assume that

$$
w=\Psi(c),
$$

where $c$ is a linear combination of monomials (4.3) in $\operatorname{Ger}(n)$ with $t=2$. 


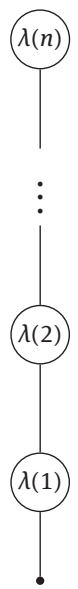

Figure 12. The brace tree $T_{\lambda}^{n}$. Here $\lambda \in S_{n}$.

Since $\Psi(c)$ is a linear combination of expressions of the form

$$
\sigma \circ \mu\left(\left(T_{\cup}+T_{\cup}^{\mathrm{opp}}\right) \otimes \mathrm{j}\left(h_{1}\right) \otimes \mathrm{j}\left(h_{2}\right)\right),
$$

where $h_{1} \in \Lambda \operatorname{Lie}\left(n_{1}\right), h_{2} \in \Lambda \operatorname{Lie}\left(n-n_{1}\right), \mu$ is the operadic multiplication, and $\sigma \in S_{n}$, the vector $\Psi(c)$ is antisymmetric with respect to the $S_{2}$ action on $\mathcal{F}^{2} V$. $(n)$ which switches the two branches originating from the lowest non-root vertex. On the other hand, the vector (4.45) is symmetric with respect to this $S_{2}$ action. Hence (4.48) follows.

We will now prove that

$$
\delta_{1}^{*}\left(T_{\|, \sigma, r}\right)-\sum_{\tau \in \mathrm{Sh}_{r, n-r}}(-1)^{|\tau|} T_{\sigma \circ \tau^{-1}}^{n} \in \delta_{0}^{*}\left(V_{\circ}^{*}(n)\right) .
$$

Then the desired statement about the vector (4.46) will follow from the graded commutativity of the shuffle product. The simple calculation shown in Figure 13 proves (4.49) in the case when $n=2$ (and $r=1$ ). This also settles the base of our induction.

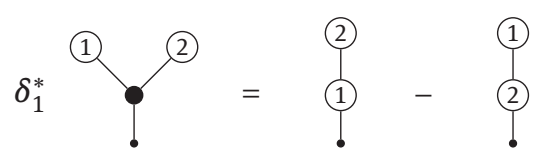

Figure 13. The proof of (4.49) in the case $n=2$.

Next, we observe that the linear combination

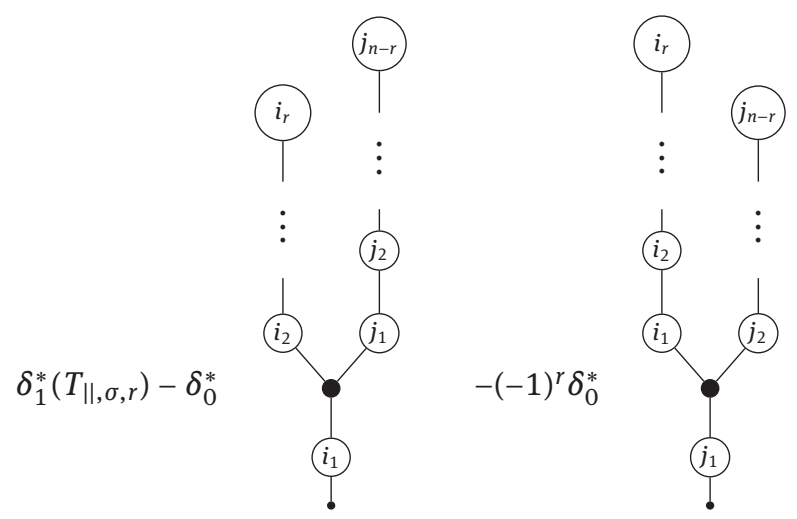


is obtained from

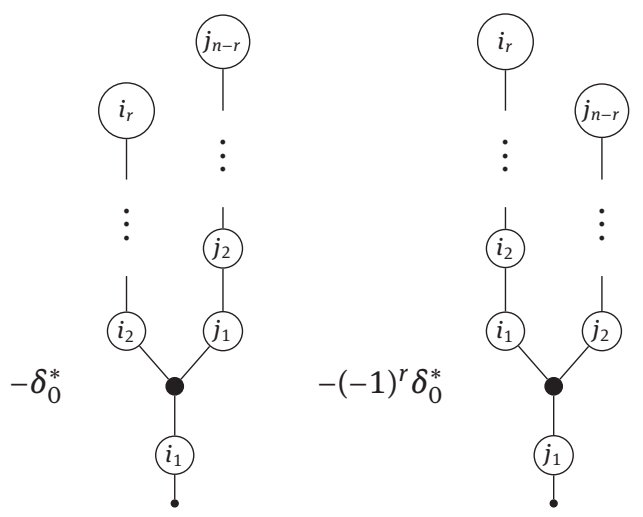

by retaining only the terms which are obtained by contracting only the edges which are adjacent to the neutral vertex and lie above this neutral vertex.

Thus the inductive step follows from the fact that the set of shuffles $\mathrm{Sh}_{r, n-r}$ splits into the disjoint union of permutations of the form

$$
\left(\begin{array}{ccccccc}
1 & 2 & \ldots & r & r+1 & \ldots & n \\
1 & \sigma(2) & \ldots & \sigma(r) & \sigma(r+1) & \ldots & \sigma(n)
\end{array}\right)
$$

with $\sigma \in S_{\{2,3, \ldots, n\}}, \sigma(2)<\sigma(3)<\cdots<\sigma(r), \sigma(r+1)<\sigma(r+2)<\cdots<\sigma(n)$, and permutations of the form

$$
\left(\begin{array}{cccccccc}
1 & 2 & \ldots & r & r+1 & r+2 & \ldots & n \\
\sigma(1) & \sigma(2) & \ldots & \sigma(r) & 1 & \sigma(r+2) & \ldots & \sigma(n)
\end{array}\right),
$$

where $\sigma$ is a bijection $\sigma:\{1,2, \ldots, r, r+2, \ldots, n\}$ to $\{2,3, \ldots, n\}$ such that $\sigma(1)<\sigma(2)<\cdots<\sigma(r)$ and $\sigma(r+2)<\sigma(r+3)<\cdots<\sigma(n)$.

Claim 4.10 is proved.

Let us recall that, for every graded vector space $^{6} \mathrm{~V}$,

$$
\operatorname{coLie}(V) \cong \operatorname{coAs}(V) / \operatorname{coAs}(V) \cdot \mathrm{sh} \operatorname{coAs}(V),
$$

where $\bullet$ sh denotes the shuffles product.

Thus Claims 4.3 and 4.10 imply that

$$
\operatorname{dim} H^{n-1}\left(\operatorname{Br}(n)^{*}\right) \leq(n-1) !
$$

and the desired inequality (4.44) follows.

\subsection{The final strokes}

Combining Claim 4.9 with inequality (4.44), we conclude that

$$
\operatorname{dim} H^{1-n}(\operatorname{Br}(n))=(n-1) !
$$

and the restriction $\left.\Psi\right|_{\Lambda L \text { ie(n) }}$ induces an isomorphism

$$
\Lambda \operatorname{Lie}(n) \cong H^{1-n}(\operatorname{Br}(n)) .
$$

Hence, due to the summary given in Section 4.5 and the second statement of Claim 4.7, it suffices to show that

$$
\operatorname{coker}\left(H^{\bullet}\left(V_{\circ}(n), \delta_{0}\right) \stackrel{H^{*}\left(\delta_{1}\right)}{\longrightarrow} H^{\bullet}\left(V_{\bullet}(n), \delta_{0}\right)\right) \cong \operatorname{Com} \odot \Lambda \operatorname{Lie}(n) / \Lambda \operatorname{Lie}(n) .
$$

6 The isomorphism (4.50) is the dual version of [18, Proposition 1.3.5]. 
The later is a consequence of (4.37), the equality $\operatorname{dim} H^{n-1}\left(\operatorname{Br}(n)^{*}\right)=(n-1)$ ! and Claim 4.10. Indeed, due to Claim 4.10 and equality $\operatorname{dim} H^{n-1}\left(\operatorname{Br}(n)^{*}\right)=(n-1)$ !, the dimension of the space $H^{*}\left(\delta_{1}^{*}\right)\left(U^{*}\right)$ should be equal to $n !-(n-1)$ !, where $U^{*}$ is the linear dual of (4.41).

On the other hand, $\operatorname{dim}(U)=n !-(n-1) !=\operatorname{dim}\left(U^{*}\right)$ and hence the restriction of $H^{*}\left(\delta_{1}^{*}\right)$ to $U^{*}$ is an isomorphism of vector spaces

$$
U^{*} \cong H^{\bullet}\left(\delta_{1}^{*}\right)\left(U^{*}\right) \subset H^{n-1}\left(V_{\circ}(n)^{*}, \delta_{0}^{*}\right) .
$$

Therefore, by duality, the composition of $H^{\bullet}\left(\delta_{1}\right)$ with the projection

$$
H^{2-n}\left(V_{\bullet}(n), \delta_{0}\right) \rightarrow U
$$

gives us an isomorphism of vector spaces

$$
H^{1-n}\left(V_{\circ}(n), \delta_{0}\right) / \operatorname{ker}\left(H^{\bullet}\left(\delta_{1}\right)\right) \cong U .
$$

Thus the desired isomorphism (4.52) follows and the proof of Theorem 4.2 is complete.

\section{A Verification of the Gerstenhaber relations}

As above, $T_{\left\{a_{1}, a_{2}\right\}}$ and $T_{a_{1} a_{2}}$ denote the following vectors in $\operatorname{Br}(2)$ :

$$
T_{\left\{a_{1}, a_{2}\right\}}:=T_{1-2}+T_{2-1}, \quad T_{a_{1} a_{2}}:=\frac{1}{2}\left(T_{\cup}+T_{\cup}^{\mathrm{opp}}\right),
$$

where $T_{1-2}, T_{2-1}, T_{\cup}$, and $T_{\cup}^{\text {opp }}$ are the brace trees shown in Figure 1.

The goal of this appendix is to prove the following statement.

Claim A.1. The vector $T_{\left\{a_{1}, a_{2}\right\}}$ satisfies the Jacobi identity

$$
T_{\left\{a_{1}, a_{2}\right\}} \circ_{1} T_{\left\{a_{1}, a_{2}\right\}}+(1,2,3)\left(T_{\left\{a_{1}, a_{2}\right\}}{ }^{\circ} 1 T_{\left\{a_{1}, a_{2}\right\}}\right)+(1,3,2)\left(T_{\left\{a_{1}, a_{2}\right\}}{ }^{\circ} 1 T_{\left\{a_{1}, a_{2}\right\}}\right)=0
$$

and the vector $T_{a_{1} a_{2}}$ fulfills these properties:

$$
\begin{aligned}
T_{a_{1} a_{2}{ }^{\circ} 1} T_{a_{1} a_{2}}-T_{a_{1} a_{2}{ }^{\circ}} T_{a_{1} a_{2}} & \in \operatorname{Im}(\delta), \\
T_{\left\{a_{1}, a_{2}\right\} \circ_{2}} T_{a_{1} a_{2}}-T_{a_{1} a_{2}{ }^{\circ} 1} T_{\left\{a_{1}, a_{2}\right\}}-(1,2)\left(T_{a_{1} a_{2}{ }^{\circ} 2} T_{\left\{a_{1}, a_{2}\right\}}\right) & \in \operatorname{Im}(\delta) .
\end{aligned}
$$

Proof. The insertion $T_{\left\{a_{1}, a_{2}\right\}}{ }^{\circ}{ }_{1} T_{\left\{a_{1}, a_{2}\right\}}$ is computed explicitly in Figure 14. It is clear that the sum over the cyclic permutations of the first term (resp. the third term) will cancel the sum over the cyclic permutations of the sixth term (resp. the forth term). Similarly, the sum over the cyclic permutations of the second term (resp. the fifth term) cancels the sum over the cyclic permutations of the seventh term (resp. the eighth term). Thus identity (A.1) holds.

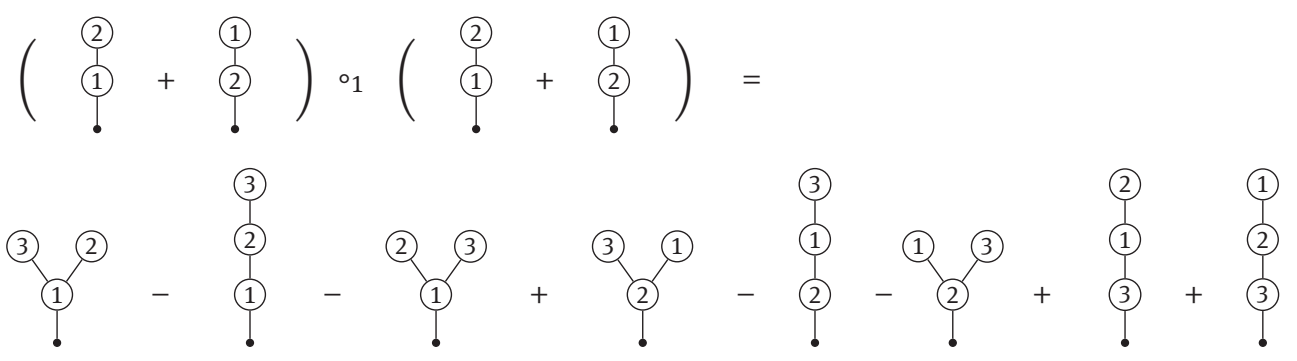

Figure 14. Computation of the vector $T_{\left\{a_{1}, a_{2}\right\}}{ }^{\circ} T_{\left\{a_{1}, a_{2}\right\}} \in \operatorname{Br}(3)$. 
A simple computation shows that

$$
\delta\left(T_{1-2}\right)=T_{\cup}-T_{\cup}^{\mathrm{opp}}
$$

Hence

On the other hand,

$$
T_{a_{1} a_{2}}=T_{\cup}-\frac{1}{2} \delta\left(T_{1-2}\right) .
$$

$$
\delta \int_{0}^{(1)}=T_{\cup \circ 1} T_{\cup}-T_{\cup} \circ 2 T_{U} \text {. }
$$

Therefore, the vector

$$
T_{a_{1} a_{2} \circ{ }_{1}} T_{a_{1} a_{2}}-T_{a_{1} a_{2} \circ{ }_{2}} T_{a_{1} a_{2}}
$$

indeed belongs to $\operatorname{Im}(\delta)$, i.e. (A.2) holds.

To prove (A.3), we denote by $T_{1-(2,3)}$ the following brace tree:

$$
T_{1-(2,3)}:=\text { (2) (1) }^{(3)}
$$

and we compute the differential $\delta\left(T_{1-(2,3)}\right)$ in Figure 15. The insertions $T_{\left\{a_{1}, a_{2}\right\}}{ }^{\circ} T_{\cup}$ and $T_{\cup}{ }^{\circ} 1 T_{\left\{a_{1}, a_{2}\right\}}$ are computed in Figures 16 and 17, respectively, and the vector $(1,2)\left(T_{\cup} \circ_{2} T_{\left\{a_{1}, a_{2}\right\}}\right)$ is shown in Figure 18.

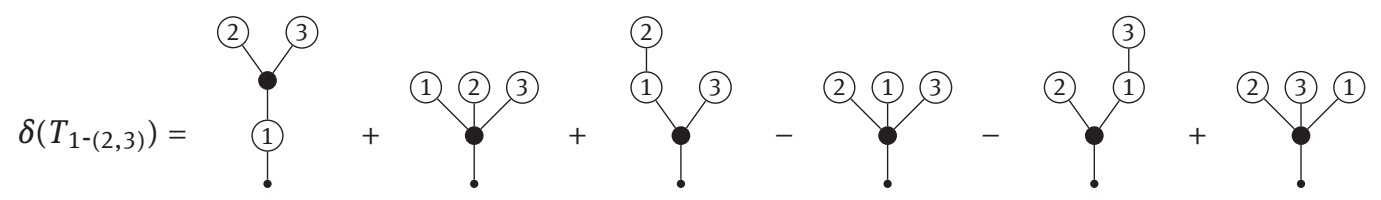

Figure 15. Computation of the differential $\delta\left(T_{1-(2,3)}\right)$.

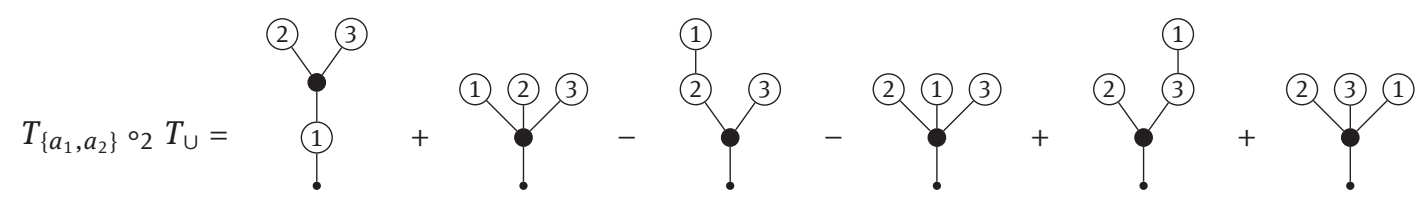

Figure 16. Computation of the insertion $T_{\left\{a_{1}, a_{2}\right\}} \circ_{2} T_{\mathrm{U}}$.

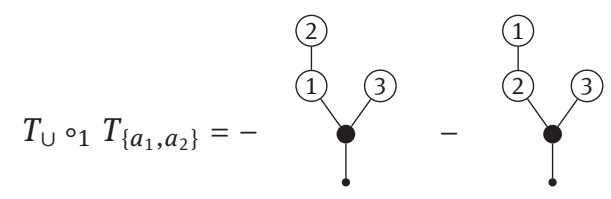

Figure 17. Computation of the insertion $T_{\cup} \circ_{1} T_{\left\{a_{1}, a_{2}\right\}}$.

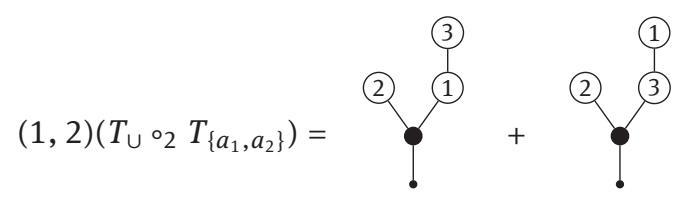

Figure 18. The vector $(1,2)\left(T_{\cup} \circ_{2} T_{\left\{a_{1}, a_{2}\right\}}\right)$.

Adding all these expressions and performing obvious cancelations, we conclude that

$$
T_{\left\{a_{1}, a_{2}\right\}} \circ_{2} T_{\cup}-T_{\cup} \circ T_{1} T_{\left\{a_{1}, a_{2}\right\}}-(1,2)\left(T_{\cup} \circ_{2} T_{\left\{a_{1}, a_{2}\right\}}\right)=\delta\left(T_{1-(2,3)}\right) .
$$

Finally, combining (A.5) with (A.6), we deduce (A.3).

Claim A.1 is proved. 


\section{B The spectral sequence for $\left(V_{0}(n), \delta_{0}\right)$ degenerates at the second page}

Let us study in a bit more detail the dual of the map j : $\Lambda$ Lie $\rightarrow B r$. In arity $n$ the dual map can be realized as a composition

$$
\operatorname{Br}^{*}(n) \rightarrow \mathcal{T}^{*}(n) \rightarrow \Lambda^{-1} \operatorname{coAs}(n) \rightarrow \Lambda^{-1} \operatorname{coLie}(n),
$$

where we use the following objects and morphisms:

- $\mathcal{T}(n) \subset \operatorname{Br}(n)$ is the graded subspace of trees without neutral vertices. (In fact, the $\mathcal{T}(n)$ assemble to form an operad whose twist is essentially $\mathrm{Br}$, cf. [5].)

- The map $\operatorname{Br}^{*}(n) \rightarrow \mathcal{T}^{*}(n)$ is the natural projection. (Concretely, it sends graphs with neutral vertices to zero.)

- The map $\Lambda^{-1} \operatorname{coAs}(n) \rightarrow \Lambda^{-1} \operatorname{coLie}(n)$ is the natural projection arising from the inclusion Lie $\rightarrow$ As. Note that we may identify $\Lambda^{-1} \operatorname{coAs}(n)$ (up to a degree shift) with the subspace of the space of words

$$
\mathbb{K}\left\langle X_{1}, \ldots, X_{n}\right\rangle
$$

in formal odd variables, each appearing exactly once. The space $\mathbb{K}\left\langle X_{1}, \ldots, X_{n}\right\rangle$ is a $\mathbb{Z}^{n}$ graded augmented commutative algebra with the shuffle product $\bullet_{\text {sh }}$ and unit the empty word. We denote by $A_{n} \subset \mathbb{K}\left\langle X_{1}, \ldots, X_{n}\right\rangle$ the augmentation ideal. The space $\Lambda^{-1}$ coLie( $\left.n\right)$ may then be identified with the degree $(1, \ldots, 1)$-subspace of the quotient

$$
A_{n} /\left(A_{n} \cdot \text { sh } A_{n}\right) .
$$

In this language, $\Lambda^{-1} \operatorname{coAs}(n) \rightarrow \Lambda^{-1} \operatorname{coLie}(n)$ is just the map induced on the degree $(1, \ldots, 1)$-subspaces of the obvious projection

$$
A_{n} \rightarrow A_{n} /\left(A_{n} \cdot \text { sh } A_{n}\right) \text {. }
$$

- The map $f: \mathcal{T}^{*}(n) \rightarrow \Lambda^{-1} \operatorname{coAs}(n) \cong A_{n}^{(1, \ldots, 1)}$ can be defined recursively as follows. If $n=1$ and $T \in \mathcal{T}^{*}(1)$ is the unique tree with one vertex labelled 1 , we set

$$
f(T)=X_{1} .
$$

If $n>1$ and $T \in \mathcal{T}^{*}(n)$ is the tree with lowest vertex $j$, having children (in this order) $T_{1}, \ldots, T_{k}$, we set recursively

$$
f(T)=X_{j}\left(f\left(T_{1}\right) \cdot \bullet_{\text {sh }} \cdots \bullet_{\text {sh }} f\left(T_{k}\right)\right) .
$$

For example, if $\lambda \in S_{n}$ and $T_{\lambda}^{n}$ is the brace tree shown in Figure 12, then

$$
f\left(T_{\lambda}^{n}\right)=X_{\lambda(1)} X_{\lambda(2)} \ldots X_{\lambda(n)} .
$$

Furthermore, if

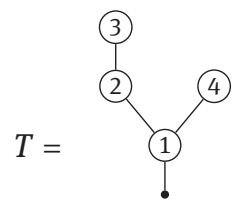

then

$$
f(T)=X_{1}\left(\left(X_{2} X_{3}\right) \cdot \operatorname{sh} X_{4}\right)=X_{1}\left(X_{2} X_{3} X_{4}-X_{2} X_{4} X_{3}+X_{4} X_{2} X_{3}\right) .
$$

The composition $g: \operatorname{Br}^{*}(n) \rightarrow \mathcal{T}^{*}(n) \stackrel{f}{\rightarrow} \Lambda^{-1} \operatorname{coAs}(n)$ appearing above is of interest in its own right. It is does not commute with the differential, i.e. $g \circ \delta^{*} \neq 0$. However, we claim that

Lemma B.1. For every brace tree $T$

$$
g \circ \delta_{0}^{*}(T)=0
$$


Proof. It is clear that we should only consider $g \circ \delta_{0}^{*}(T)$ for a brace tree $T$ with exactly one neutral vertex which is not in the lowest possible position.

Up to an overall sign factor, the differential $\delta_{0}^{*}$ turns the branch

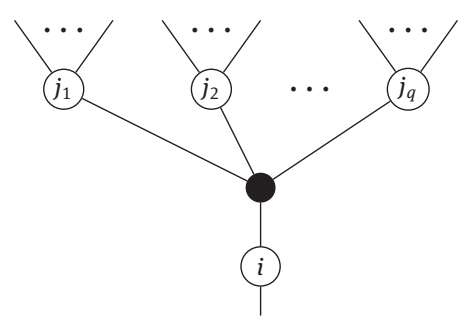

into the linear combination
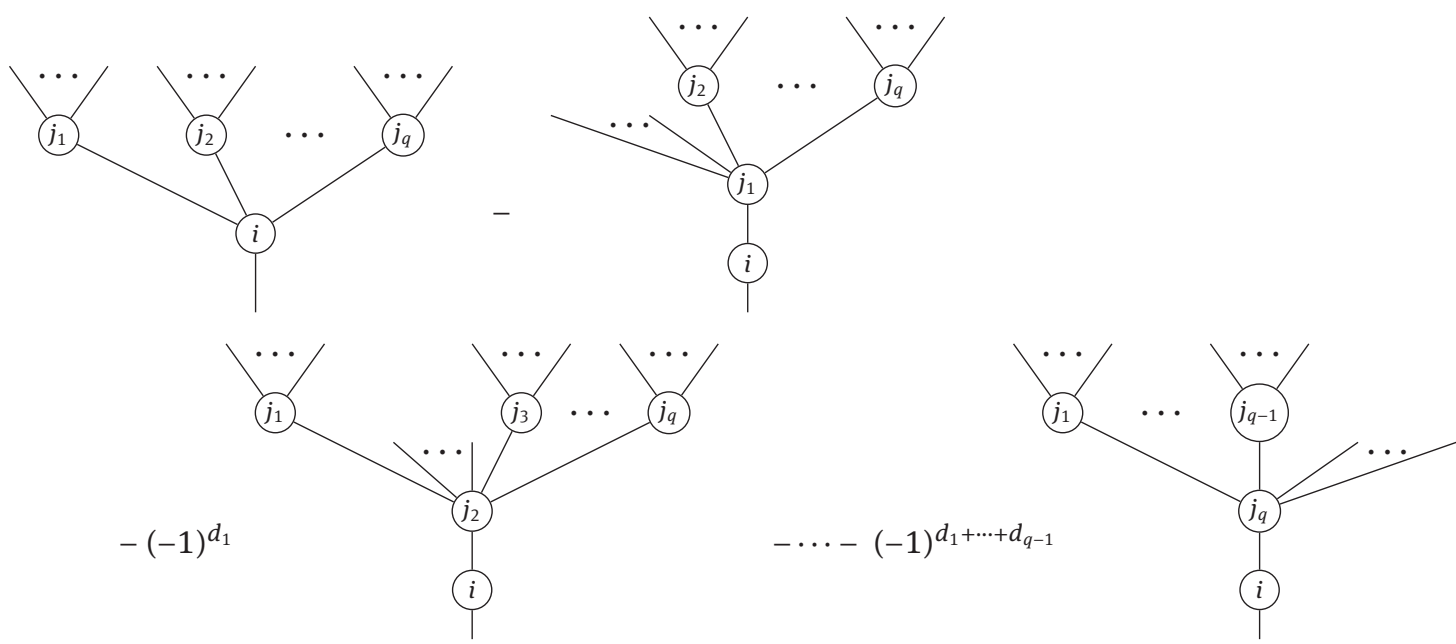

where $d_{k}$ is the degree of the branch which originates from the neutral vertex and contains vertex $j_{k}$.

Therefore $g \circ \delta_{0}^{*}(T)$ contains this expression

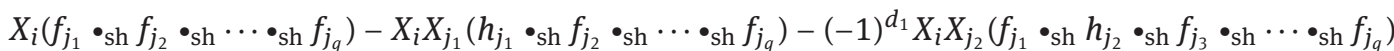

$$
\begin{aligned}
& -\cdots-(-1)^{d_{1}+d_{2}+\cdots+d_{q-1}} X_{i} X_{j_{q}}\left(f_{j_{1}} \cdot \operatorname{sh} \cdots \cdot \bullet_{\text {sh }} f_{j_{q-1}} \cdot \operatorname{sh} h_{j_{q}}\right)
\end{aligned}
$$

as a factor. Here $f_{j_{k}}$ is the value of $f$ on the branch which originates at the neutral vertex and contains vertex $j_{k}$, while

$$
h_{j_{k}}=f\left(b_{j_{k} 1}\right) \cdot \bullet_{\mathrm{sh}} f\left(b_{j_{k}}\right) \cdot \bullet_{\mathrm{sh}} \cdots \cdot \bullet_{\mathrm{sh}} f\left(b_{j_{k} r_{k}}\right),
$$

where $b_{j_{k}}$ is the $t$-th branch which originates from vertex $j_{k}$.

Using the definition of the shuffle product, it is easy to see that the expression (B.1) is zero.

Thus the lemma follows.

Remark B.2. Let us observe that the map $g \circ \delta_{1}^{*}$ has the following nice combinatorial description: If $T \in \mathrm{Br}^{*}(n)$ is a brace tree, then $g \circ \delta_{1}^{*}(T)=0$ unless $T$ has exactly one neutral vertex, which is the lowest vertex. In this case

$$
g \circ \delta_{1}^{*}(T)=f\left(T_{1}\right) \cdot \text { sh } \cdots \cdot \bullet_{\text {sh }} f\left(T_{k}\right),
$$

where $T_{1}, \ldots, T_{k}$ are the branches which originate at the neutral vertex.

On the other hand, Lemma B.1 implies that $g \circ \delta^{*}=g \circ \delta_{1}^{*}$. Thus $g \circ \delta^{*}(T)=0$ unless $T$ has exactly one neutral vertex, which is the lowest vertex and, in this case,

$$
g \circ \delta^{*}(T)=f\left(T_{1}\right) \cdot \text { sh } \cdots \cdot \text { sh } f\left(T_{k}\right) .
$$

Let us now consider the dual cochain complex $\left(V_{\bullet}(n)^{*}, \delta_{0}^{*}\right)$ and construct a set of vectors in the top degree $n-2$ which will play an important role. 


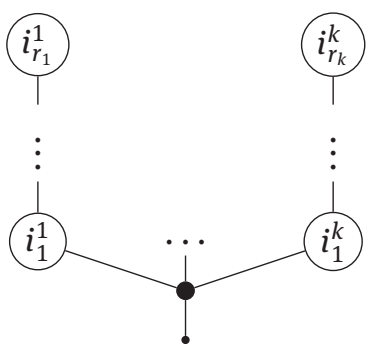

Figure 19. The brace tree $T_{r_{1}, \ldots, r_{k}}^{\sigma}$.

Let $k$ be an integer $\geq 2$ and $\left(r_{1}, r_{2}, \ldots, r_{k}\right)$ a tuple of positive integers such that $r_{1}+r_{2}+\cdots+r_{k}=n$. For every such tuple, we consider a brace trees $T_{r_{1}, \ldots, r_{k}}^{\sigma}$ shown in Figure 19, where $\sigma$ is a permutation in $S_{n}$

$$
\sigma=\left(\begin{array}{cccccccccccc}
1 & 2 & \ldots & r_{1} & r_{1}+1 & \ldots & r_{1}+r_{2} & \ldots & \ldots & n-r_{k}+1 & \ldots & n \\
i_{1}^{1} & i_{2}^{1} & \ldots & i_{r_{1}}^{1} & i_{1}^{2} & \ldots & i_{r_{2}}^{2} & \ldots & \ldots & i_{1}^{k} & \ldots & i_{r_{k}}^{k}
\end{array}\right)
$$

which satisfies the properties ${ }^{7}$

$$
i_{1}^{m}=\min \left\{i_{1}^{m}, i_{2}^{m}, \ldots, i_{r_{m}}^{m}\right\} \quad \text { for all } m, \quad i_{1}^{1}<i_{1}^{2}<\cdots<i_{1}^{k} .
$$

Moreover, we set

$$
Y_{r_{1}, \ldots, r_{k}}^{\sigma}=\frac{1}{k !} \sum_{\tau \in S_{k}} \tau_{*}\left(T_{r_{1}, \ldots, r_{k}}^{\sigma}\right),
$$

where $\tau_{*}$ rearranges the $k$ branches of $T_{r_{1}, \ldots, r_{k}}^{\sigma}$ originating from the neutral vertex with the appropriate sign factor. For example,

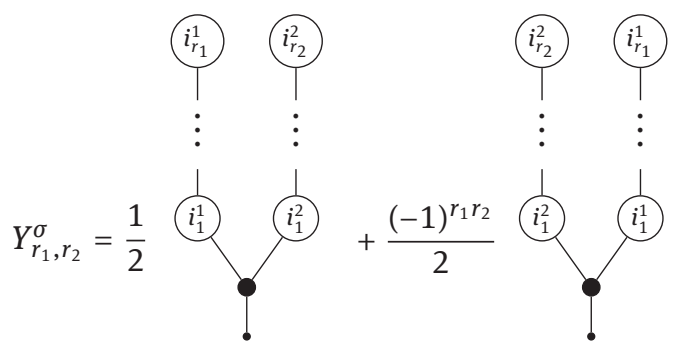

We denote by $\Xi$ the set of all such vectors $Y_{r_{1}, \ldots, r_{k}}^{\sigma}$ for all $k \geq 2$, all tuples $\left(r_{1}, r_{2}, \ldots, r_{k}\right), r_{1}+\cdots+r_{k}=n$, and all permutations $\sigma$ satisfying (B.4). Due to the theorem about the cyclic decomposition of a permutation, it is clear that $\Xi$ has $n !-(n-1)$ ! elements. Moreover, the subset $\Xi \subset V_{\bullet}(n)^{*}$ is linearly independent.

Since every vector $Y_{r_{1}, \ldots, r_{k}}^{\sigma}$ is in the top degree of $\left(V_{\bullet}(n)^{*}, \delta_{0}^{*}\right)$, it is automatically a cocycle in this complex.

Let us prove that

Claim B.3. Every non-trivial linear combination of vectors in $\Xi$ is a non-trivial cocycle in $\left(V \cdot(n)^{*}, \delta_{0}^{*}\right)$.

Proof. To prove this claim, we need Lemma B.1 and Remark B.2.

Let us, first, prove that the map

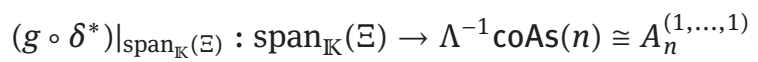

is injective. Indeed, by Remark B.2 and the symmetry of the shuffle product, we have

$$
g \circ \delta^{*}\left(Y_{r_{1}, \ldots, r_{k}}^{\sigma}\right)=g \circ \delta^{*}\left(T_{r_{1}, \ldots, r_{k}}^{\sigma}\right)=\left(X_{i_{1}^{1}} \ldots X_{i_{r_{1}}^{1}}\right) \bullet \operatorname{sh} \ldots \bullet \operatorname{sh}\left(X_{i_{1}^{k}} \ldots X_{i_{r_{k}}^{k}}\right) .
$$

Using the identification between $\Lambda^{-1} \operatorname{coLie}(n)$ and the degree $(1, \ldots, 1)$-subspace of $A_{n} /\left(A_{n} \bullet_{\text {sh }} A_{n}\right)$, it is easy to see that $g \circ \delta^{*}$ gives us a surjective map from $\operatorname{span}_{\mathbb{K}}(\Xi)$ to the degree $(1, \ldots, 1)$-subspace of $A_{n} \bullet_{\mathrm{sh}} A_{n}$. Since both $\operatorname{span}_{\mathbb{K}}(\Xi)$ and the degree $(1, \ldots, 1)$-subspace of $A_{n} \bullet$ sh $A_{n}$ have the same dimension $n !-(n-1)$ !, we conclude that (B.6) is indeed injective.

7 In particular, $i_{1}^{1}$ is necessarily 1. 
Let us consider a vector $v \in \operatorname{span}_{\mathbb{K}}(\Xi)$ and assume that $v=\delta_{0}^{*}(w)$ for some $w \in V$. $(n)^{*}$. Using Lemma B.1, we conclude that

$$
g\left(\delta^{*} v\right)=g\left(\delta^{*} \delta_{0}^{*} w\right)=-g\left(\delta_{0}^{*} \delta^{*} w\right)=0 .
$$

Thus, since (B.6) is injective, we conclude that $v=0$ and the desired claim follows.

With these preparations we are now ready to prove the following statement left open above.

Lemma B.4. The spectral sequence arising in Section 4.4 degenerates at the second page.

Proof. According to Claim 4.5, $E_{2} V .(n)$ splits (as the graded vector space) into the direct sum

$$
\operatorname{Com} \odot \Lambda \operatorname{Lie}(n) / \Lambda \operatorname{Lie}(n) \oplus \mathbf{S}(\Lambda \operatorname{Com} \odot \Lambda \operatorname{Lie}(n) / \Lambda \operatorname{Lie}(n)) .
$$

It is easy to see that every vector in the summand

$$
U:=\mathbf{s}(\Lambda \operatorname{Com} \odot \Lambda \operatorname{Lie}(n) / \Lambda \operatorname{Lie}(n))
$$

has degree $2-n$, while the summand

$$
X:=\operatorname{Com} \odot \Lambda \operatorname{Lie}(n) / \Lambda \operatorname{Lie}(n)
$$

lives in degrees $2-n \leq \bullet \leq 0$.

We also know that every vector in (B.8) can be represented by a genuine cocycle in $\operatorname{Br}(n)$. Thus the restriction of all higher differentials $d_{r},(r \geq 2)$ to the subspace (B.8) is zero and it remains to show that the restriction of $d_{r}$ for $r \geq 2$ to (B.7) is also zero.

To prove this statement, we pass to the obvious dual version of Claim 4.5, which says that

$$
E_{2} V \cdot(n)^{*} \cong X^{*} \oplus U^{*},
$$

where $X^{*}$ is the kernel of the map $\operatorname{Ger}(n)^{*} \rightarrow \Lambda \operatorname{Lie}(n)^{*}$ and $U^{*}$ is the linear dual of (B.7).

The advantage of passing to the dual complex is that $U^{*}$ lives in the top degree $n-2$ of the cochain complex $\left(V_{\bullet}(n)^{*}, \delta_{0}^{*}\right)$. So all vectors in $U^{*}$ can be represented by genuine cocycles in $\left(V_{\bullet}(n)^{*}, \delta_{0}^{*}\right)$. Moreover, the first (potentially) non-zero differential $d_{r}^{*}, r \geq 2$, may only send vectors in $X^{*}$ of degree $n-3$ to vectors in $U^{*}$ :

$$
\left(X^{*}\right)^{n-3} \rightarrow U^{*}=\left(U^{*}\right)^{n-2} .
$$

Using the explicit representatives of vectors in $X$ (see (B.8)) and the $S_{k}$-symmetry of $Y_{r_{1}, \ldots, r_{k}}^{\sigma}$, we see that the evaluation of every vector $Y_{r_{1}, \ldots, r_{k}}^{\sigma}$ on representatives of vectors in $X$ is zero. Thus all elements in $\Xi$ represent vectors in $U^{*}$.

By Claim B.3, the cohomology classes of $\Xi$ in $H^{n-2}\left(V \cdot(n)^{*}, \delta_{0}^{*}\right)$ span a subspace of dimension $n !-(n-1)$ !. Thus, since $U^{*}$ also has dimension $n !-(n-1)$ ! and the only component of the first potentially non-zero $d_{r}^{*}$ is (B.9), we conclude that $\operatorname{dim}\left(E_{\infty} V_{\bullet}(n)\right) \geq \operatorname{dim}\left(E_{2} V_{\bullet}(n)\right)$.

Lemma B.4 is proved.

Acknowledgment: We would like to thank anonymous referees for their useful comments and suggestions.

Funding: Vasily Dolgushev has been supported partially by NSF grants DMS-1161867 and DMS-1501001 and Thomas Willwacher has been supported partially by the Swiss National Science foundation, grant 200021_150012, and the SwissMAP NCCR funded by the Swiss National Science foundation.

\section{References}

[1] C. Berger and B. Fresse, Combinatorial operad actions on cochains, Math. Proc. Cambridge Philos. Soc. 137 (2004), no. 1, 135-174.

[2] D. Calaque and T. Willwacher, Triviality of the higher formality theorem, Proc. Amer. Math. Soc. 143 (2015), no. 12, 5181-5193.

[3] P. Deligne, Letter to V. Drinfeld, M. Gerstenhaber, J. P. May, V. Schechtman and J. Stasheff, unpublished, 1993. 
[4] V. A. Dolgushev and C. L. Rogers, Notes on algebraic operads, graph complexes, and Willwacher's construction, in: Mathematical Aspects of Quantization, Contemp. Math. 583, American Mathematical Society, Providence (2012), 25-145.

[5] V. A. Dolgushev and T. H. Willwacher, Operadic Twisting - with an application to Deligne's conjecture, J. Pure Appl. Algebra 219 (2015), no. 5, 1349-1428.

[6] V. G. Drinfeld, On quasitriangular quasi-Hopf algebras and on a group that is closely connected with $\mathrm{Gal}(\overline{\mathbb{Q}} / \mathbb{Q})$ (in Russian), Algebra i Analiz 2 (1990), no. 4, 149-181.

[7] M. Gerstenhaber, The cohomology structure of an associative ring, Ann. of Math. (2) 78 (1963), 267-288.

[8] E. Getzler, Cartan homotopy formulas and the Gauss-Manin connection in cyclic homology, in: Quantum Deformations of Algebras and Their Representations (Rehovot 1992), Israel Math. Conf. Proc. 7, Bar-Ilan University, Ramat Gan (1993), 65-78.

[9] E. Getzler and J. D. S. Jones, Operads, homotopy algebra and iterated integrals for double loop spaces, preprint (1994), https://arxiv.org/abs/hep-th/9403055.

[10] R. M. Kaufmann, Operads, moduli of surfaces and quantum algebras, in: Woods Hole Mathematics, Ser. Knots Everything 34, World Scientific, Hackensack (2004), 133-224.

[11] R. M. Kaufmann, On several varieties of cacti and their relations, Algebr. Geom. Topol. 5 (2005), 237-300.

[12] R. M. Kaufmann, On spineless cacti, Deligne's conjecture and Connes-Kreimer's Hopf algebra, Topology 46 (2007), no. 1, 39-88.

[13] R. M. Kaufmann and R. Schwell, Associahedra, cyclohedra and a topological solution to the $A_{\infty}$-Deligne conjecture, $A d v$. Math. 223 (2010), no. 6, 2166-2199.

[14] M. Kontsevich, Operads and motives in deformation quantization, Lett. Math. Phys. 48 (1999), no. 1, 35-72.

[15] M. Kontsevich and Y. Soibelman, Deformations of algebras over operads and the Deligne conjecture, in: Conférence Moshé Flato 1999: Quantization, Deformation, and Symmetries (Dijon 1999), Math. Phys. Stud. 21, Kluwer, Dordrecht (2000), 255-307.

[16] P. Lambrechts and I. Volić, Formality of the little N-disks operad, Mem. Amer. Math. Soc. 230 (2014), no. 1079, 1-116.

[17] J.-L. Loday, Cyclic Homology, Grundlehren Math. Wiss. 301, Springer, Berlin, 1992.

[18] J.-L. Loday and B. Vallette, Algebraic Operads, Grundlehren Math. Wiss. 346, Springer, Berlin, 2012.

[19] J. E. McClure and J. H. Smith, A solution of Deligne's Hochschild cohomology conjecture, in: Recent Progress in Homotopy Theory (Baltimore 2000), Contemp. Math. 293, American Mathematical Society, Providence (2002), 153-193.

[20] J. E. McClure and J. H. Smith, Multivariable cochain operations and little $n$-cubes, J. Amer. Math. Soc. 16 (2003), no. 3 , 681-704.

[21] P. Ševera and T. Willwacher, Equivalence of formalities of the little discs operad, Duke Math. J. 160 (2011), no. 1, 175-206.

[22] D. Tamarkin, Another proof of M. Kontsevich formality theorem, preprint (1998), https://arxiv.org/abs/math/9803025.

[23] D. Tamarkin, Formality of chain operad of little discs, Lett. Math. Phys. 66 (2003), no. 1-2, 65-72.

[24] D. Tamarkin, What do DG categories form?, Compos. Math. 143 (2007), no. 5, 1335-1358.

[25] C. A. Weibel, An Introduction to Homological Algebra, Cambridge Stud. Adv. Math. 38, Cambridge University Press, Cambridge, 1994. 